\title{
Ostracod Biostratigraphy of the Kimmeridgian in Northern and Western Europe
}

\author{
By \\ Ole Bruun Christensen \& Thomas I. Kilenyi
}

\author{
Dansk sammendrag \\ Ostracoder og biostratigrafi \\ i Nord- og Vesteuropas kimmeridgien
}

I kommission hos

C. A. REITZELS FORLAG

KØBENHAVN 1970 
D A N M A R K S II. R $Æ$ K K E. N R. 95

Geological Survey of Denmark. II. Series. No. 95

\title{
Ostracod Biostratigraphy of the Kimmeridgian in Northern and Western Europe
}

\author{
By \\ Ole Bruun Christensen \& Thomas I. Kilenyi
}

Dansk sammendrag:

Ostracoder og biostratigrafi

i Nord- og Vesteuropas kimmeridgien

I kommission hos

C. A. REITZELS FORLAG K Ø B E N H A V N 1970 
With 4 plates

Med 4 tavler

ISBN 8742106109

Printed in Denmark by Andelsbogtrykkeriet in Odense 


\section{CONTENTS}

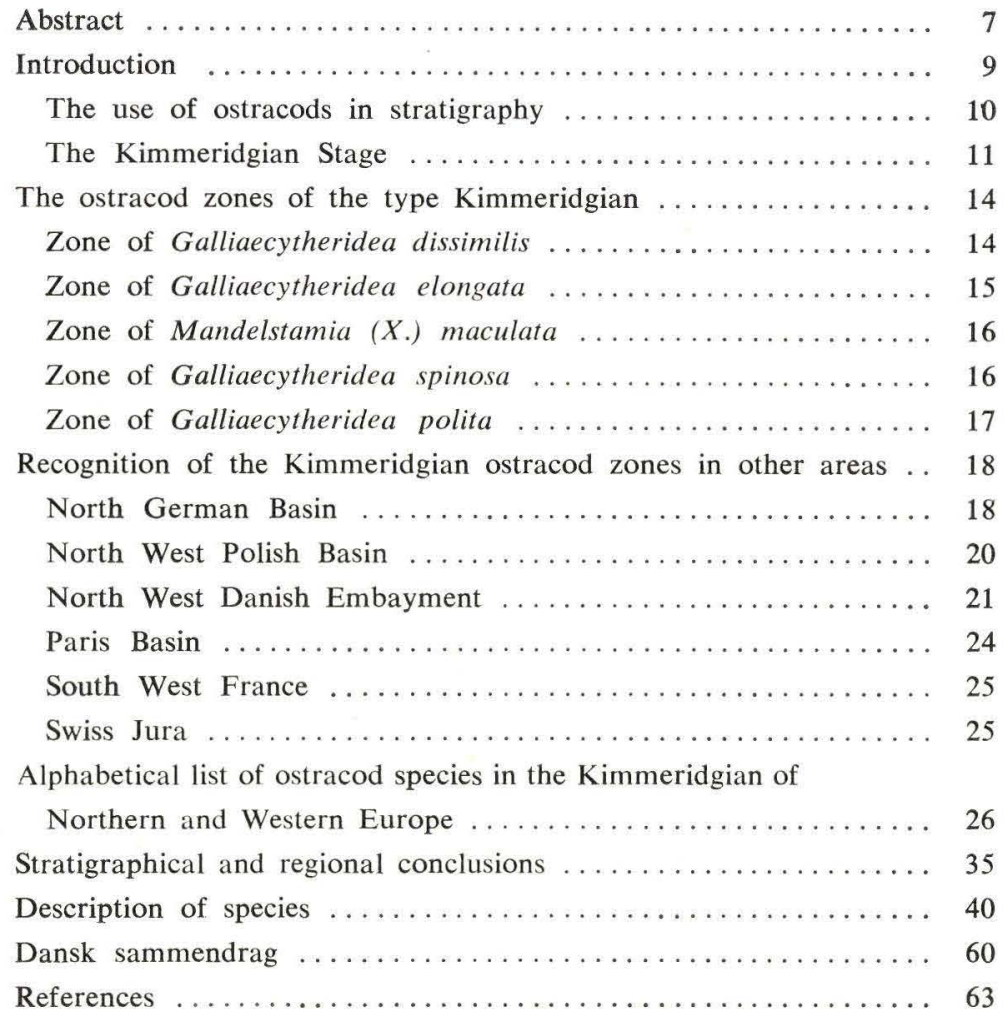

Plates I-IV 


\section{PREFACE}

This paper has been prepared during periods the authors spent together in England and Denmark. We are very grateful for financial assistance with travelling expenses and publication contributed by the Director of the Geological Survey of Denmark (DGU), Dr. Ole Bertelsen, by Sir John Cass College and by the Inner London Education Authority.

We would like to acknowledge the kind hospitality of Dr. JoHN W. NEALE of the Department of Geology, University of Hull, who made it possible for us to examine the Dorset type material. We would also like to thank Dr. R. H. BAte, British Museum (Natural History) who lent us type material from the Portland Sand of Dorset.

Finally we must express our appreciation to Mrs. Rigmor Borg, Head of the Drawing Office of the D.G.U., to Mr. Chr. Westergaard, and to Mr. O. Neergaard Rasmussen, D.G.U. photographers, for their invaluable assistance with the illustrations.

London, 6th February, 1970.

Ole Bruun Christensen, Geological Survey of Denmark,

Thomas I. Kilenyi, Copenhagen. Department of Geology, Sir John Cass College, London. 


\begin{abstract}
The chronostratigraphical type profile of the Kimmeridgian of Dorset is subdivided biostratigraphically into five ostracod zones. Six other areas in Northern and Western Europe with Kimmeridgian ostracods are examined. The species are listed, counted, and examined in relation to the zones of the type Kimmeridgian.

In the Lower Kimmeridgian the ostracod faunas bear the impression of a relatively uniform composition between the examined areas. In the Upper Kimmeridgian two different faunal regions are developed. In the North Sea Basin in the North Western Danish Embayment and in Dorset relatively uniform faunas occur, separated from other, again rather uniform faunas occuring in the Mid-European Region, from Northwestern Poland and Scania to the Paris Basin.

Stratigraphically important are species of the genera Galliaecytheridea, Mandelstamia, and Macrodentina. 19 species are given diagnoses. Two new subgenera and seven new species are established.
\end{abstract}




\section{INTRODUCTION}

The aim of this paper is to attempt to utilize ostracod faunas for biostratigraphical correlations in the Kimmeridgian of Northern and Western Europe. During the last two decades a considerable amount of information has accumulated about the Kimmeridgian ostracods of this area, and although the various areas are documented to varying degrees it seems possible at this stage to give an outline of the biostratigraphy. Seven areas of Northern and Western Europe are considered (fig. 1):

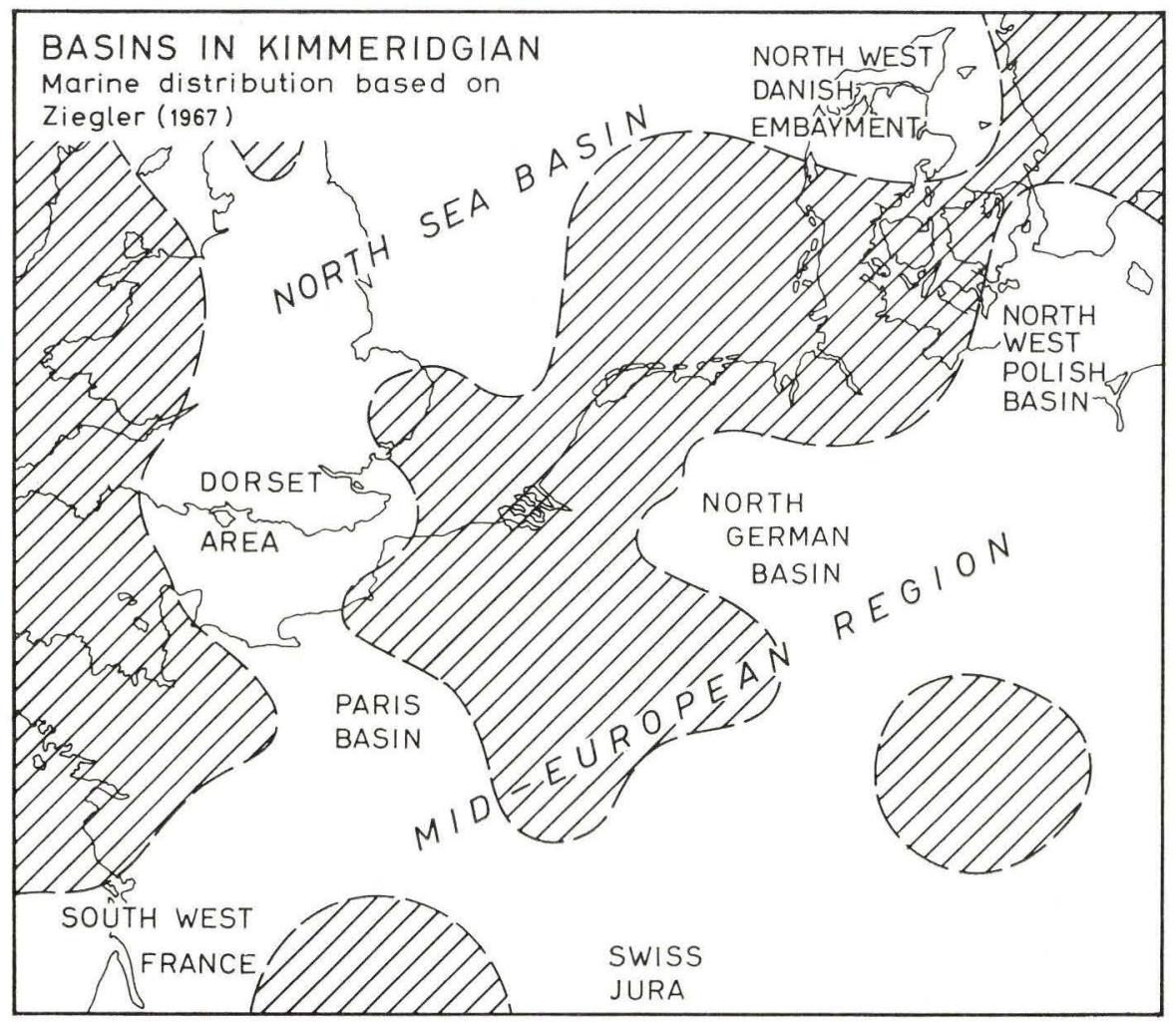

Fig. 1. 
1 Dorset

2 North German Basin

3 North West Polish Basin

4 North West Danish Embayment

5 Paris Basin

6 South West France (Ile d'Oleron)

7 Swiss Jura

A full list of ostracods described from these areas is given on p. 27. The list includes some, but not all the forms treated with open nomenclature and nomina nuda. Some of the most important species are given full synonymy and diagnosis. In addition six species are described as new and species mentioned with open nomenclature will be described in the near future (Bruun Christensen MS).

The type section of the stage (the Dorset Kimmeridge Clay) is divided into five distinct ostracod zones and the other areas are correlated with the type section. The degree of similarity between the faunas of the different areas is also examined and its palaeogeographical implications discussed.

Throughout the paper the term "Kimmeridgian Stage" is used in the English sense.

\section{THE USE OF OSTRACODS IN STRATIGRAPHY}

Ostracods have been used in stratigraphy with varying degree of success in the past. Species of the fresh-water genus Cypridea for example furnish many good and precise local range and assemblage zones in the Upper Jurassic and Lower Cretaceous Purbeck-Wealden type deposits. Marine ostracods are, however, on the whole relatively slowly evolving organisms and their geographical distribution is strongly influenced by facies - as is the case with most benthonic fossils. Biostratigraphy based on marinebrackish ostracods, therefore, cannot equal the precision afforded by the use of quickly evolving as well as quickly migrating planktonic and nektonic organisms (Ammonoidea etc.) The great advantage of ostracods in biostratigraphy is the wealth of marine and marine-brackish species which are not effected by a certain deviation from the standard marine salinity. This is particularly important in the Upper Kimmeridgian where in the areas considered - with the exception of Dorset - ammonite faunas are scarce or totally absent. Furthermore, in the study of material from boreholes one is restricted to microfossils.

As stated above marine-brackish ostracods have only rarely been used as indicators of range zones. The five ostracod zones designated in the type section are used in the sense of assemblage zones. 


\section{THE KIMMERIDGIAN STAGE}

The type section of the Kimmeridgian Standard Stage is the formation of Kimmeridge Clay of Dorset. The formation was first recognized and named by Webster (1816) as "Kimmeridge Strata", followed by FitTon (1836) who first used the term "Kimmeridge Clay". The maximum thickness of the Kimmeridge Clay in Dorset is approximately 500 metres (ARKell 1956) of which about 325 metres is exposed in the main type section (Kimmeridge Bay-Chapman's Pool). The lowermost parts are exposed further to the West in the neighbourhood of Osmington Mills where the thickness of the formation is somewhat reduced.

Lithologically the Kimmeridge Clay is a very uniform formation, consisting of dark shales and clays with occasional hard cementstone bands which are quite persistent horizontally and may be used as reference horizons locally. The shales and clays contain abundant ammonites (mostly flattened or crushed) and in certain horizons brachiopods and bivalves may be quite common. The hitherto used ammonite zonation of the Kimmeridge Clay (ARKELL 1933, 1947) is now being revised and the zonal scheme used in fig. 2 is based on Ziegler (1962), Cope (1967, 1968), CASEY (1967) and Cope, Hallam, Thorrens et al. (1969).

When D'Orbigny (1842-1849) introduced his Jurassic stages he took the Kimmeridge Clay of Dorset as the type section of his "Etage Kimmeridgien" and the following Portland Sand and Portland Stone formations as the basis of his "Portlandien". Due to his misidentification of Gravesia (which occurs within the Kimmeridge Clay) from calcareous deposits in Germany and France as the large titanitids of the English Portland beds a dual and very misleading usage of the term Kimmeridgian Stage had arisen. In the British literature the Kimmeridgian Stage extends up to the Albani Zone (base of the formation of Portland Sand in Dorset) whereas on the continent beds containing Gravesia are included in the "Portlandian" Stage. Thus the Lower Kimmeridgian of Dorset became synonymous with the whole of the Kimmeridgian of the continent and the Upper Kimmeridgian of the British terminology with the Lower Portlandian of the French and German authors. This dual and undesirable usage of the term still persists in the modern literature. As the type section of the stage is the Dorset Kimmeridge Clay throughout this paper the Kimmeridgian Stage is based on the whole formation.

ArKell (1933) divided the Kimmeridge Clay (and thus the Kimmeridgian Stage) into a Lower and Upper division, taking the "Yellow Ledge Stone Band" as the line of division. In this sense he included the Gravesia Zone into the lower division. Later ArKell (1947, p. 66) follows the same classification but in the same paper (table on p. 67) he includes the Gravesia Zone in the Upper Kimmeridge Clay. In his "Jurassic Geology of the World" 


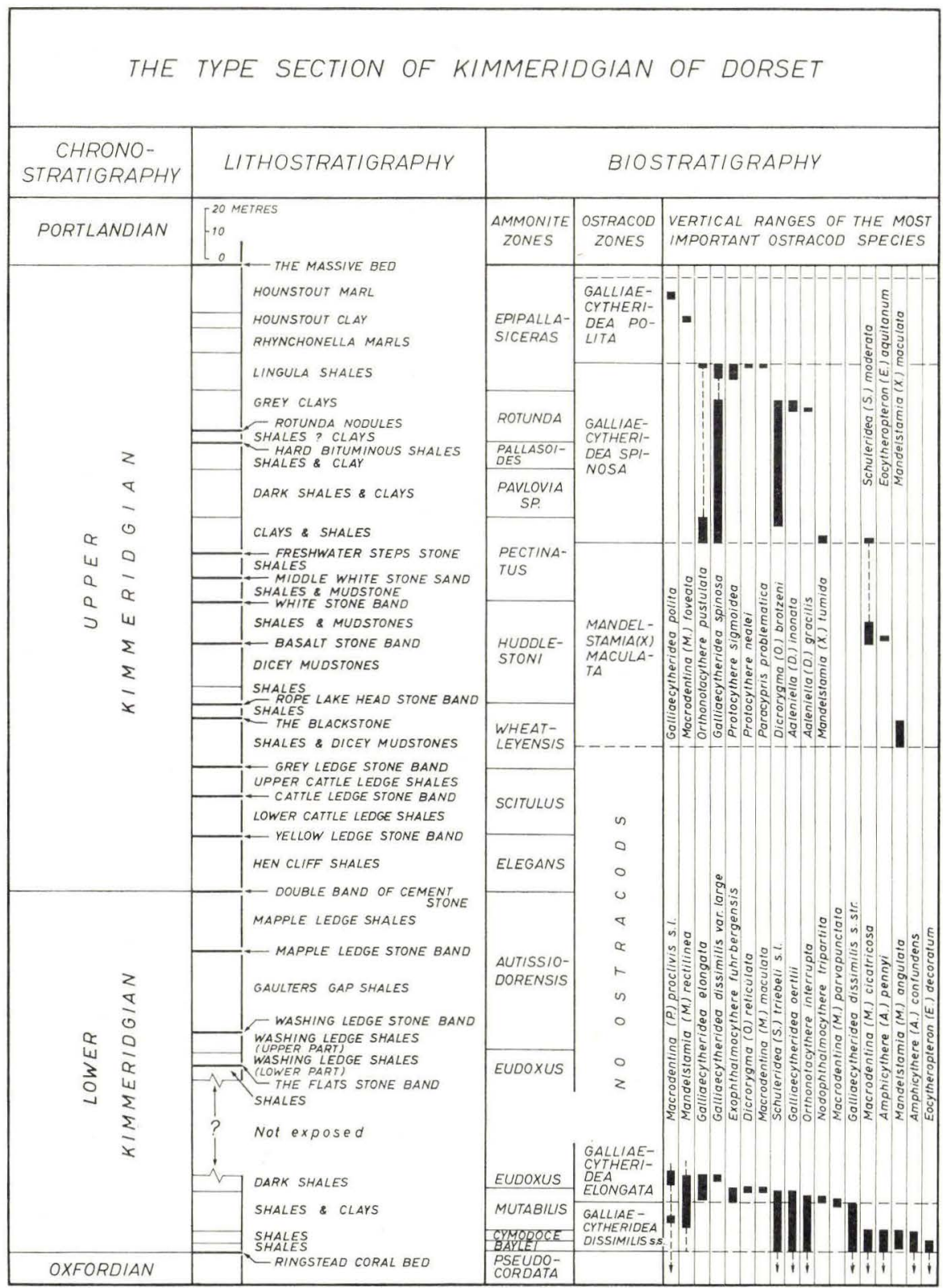

Fig. 2 .

Arkell (1956) introduces a threefold division of the formation, and the stage, the Gravesia Zone forming the base of the Middle division. Cope (1967, p. 4, footnote) states that "the Middle Kimmeridgian of Arkell (1956, p. 21) has ... no real standing", and draws the boundary between the 
Lower and Upper Kimmeridgian at the top of the Aulacostephanus autissiodorensis Zone (base of the "Hen Cliff Shales", see fig. 2 p. 12). This has been used as the "Kimmeridgian/Portlandian" border on the continent. The vertical distribution of ostracod faunas also suggests that the twofold division of the stage is more practicable. 


\section{THE OSTRACOD ZONES OF THE TYPE KIMMERIDGIAN}

Five ostracod zones are here stated for the Dorset Kimmeridge Clay, two in the lower and three in the upper division. Unfortunately there is a gap in the zonal scheme between the two lowermost and the three uppermost zones (covering the ammonite zones from the upper Eudoxus to near the top of the Scitulus Zone) due to the lack of the ostracods in these beds.

\section{ZONE OF GALLIAECYTHERIDEA DISSIMILIS O E R T L I S. STR.}

This Zone extends from the base of the Kimmeridge Clay (top of Ringstead Coral Bed) to the middle of the Mutabilis Zone. It is characterized by the dominance of $G$. dissimilis OERTLI s. str. and the top of the Zone is marked by the disappearance of this species. Higher up in the Lower Kimmeridge Clay (Eudoxus Zone) there are occurrences of a similar form (KILENYI 1969, plate 24, figs. 15-20) which are distinctly larger than the typical $G$. dissimilis OertLi $s$. str. and show a characteristic postero-ventral bulge. From stratigraphically higher levels in the Lower Kimmeridgian of Denmark occur specimens of Galliaecytheridea dissimilis OERTLI $s$. 1. with an extremely accentuated posteroventral extension. Thus these larger specimens from the Dorset Kimmeridge Clay are transitional between $G$. dissimilis OerTLI s. str. and the above mentioned Danish specimens and probably represent a transition subspecies.

The base of the Zone is not established in the Kimmeridgian Clay as the zonal index, G. dissimilis Oertli s. str. extends down to the Cautisnigrae Zone (Upper Oxfordian) in Dorset and elsewhere. Mandelstamia (M.) angulata KILENYI makes its first appearence in the basal Kimmeridgian, but as it has not been found elsewhere, not much stratigraphical reliance can be placed upon it. Very important is, however, the negative evidence of Lophocythere multicostata OERTLI, a species that dies out in the highest Oxfordian and indeed no species of this genus crosses the Oxfordian-Kimmeridgian boundary. 
The following species occur in the Zone (species marked $*$ are confined to the Zone):

* Galliaecytheridea dissimilis Oertur $s$. str. Amphicythere (A.) confundens OERTLI Amphicythere (A.) pennyi KILENYI

* Amphicythere (A.) sphaerulata KILENYI Cytherella recta SHARAPOVA Eocytheropteron (E.) decoratum (SCHMIDT)

Galliaecytheridea confundens KILENYI

Galliaecytheridea dorsetensis nov. sp.

Galliaecytheridea fragilis KILENYI

* Galliaecytheridea gracilis GLASHOFF

Galliaecytheridea postrotunda OERTLI

Galliaecytheridea punctata KILENYI

Macrodentina (M.) cicatricosa MALZ

Macrodentina (P.) parvapunctata KILENYI

Macrodentina (P.) proclivis Malz s. l.

* Mandelstamia (M.) angulata KILENYI

Mandelstamia (M.) rectilinea MALZ

Orthonotacythere interrupta TRIEBEL

* Paracypris sp. C OertLI

Procytheropteron sp. 1 KILENYI

* Protocythere rodewaldensis (KLINGLER)

* Rectocythere (L.) sp. 1 (KILENYI)

Schuleridea triebeli (Steghaus)

2. ZONE OF GALLIAECYTHERIDEAELONGATA

\section{K I L E N Y I}

The base of the Zone lies approximately in the Mutabilis Zone. It is extremely well and sharply defined by the first appearance of the zonal index, $G$. elongata KILENYI, Exophthalmocythere fuhrbergensis STEGHAUS and Nodophthalmocythere tripartita MALZ.

The following species occur in the Zone (species marked * are confined to the Zone):

* Galliaecytheridea elongata KILENYI

Cytherelloidea paraweberi OERTLI

Cytherelloidea weberi Steghaus

* Dicrorygma (O.) reticulata BRUUn Christensen

* Exophthalmocythere fuhrbergensis Steghaus

Galliaecytheridea confundens KILENYI 
* Galliaecytheridea dissimilis OeRTLI (large var.)

Galliaecytheridea dorsetensis nov. sp. (confined to the lower part of the zone)

* Galliaecytheridea cf. mandelstami (Luubimova)

* Galliaecytheridea sp. 1 KILENYI

* Macrodentina (M.) maculata MALz

Macrodentina (P.) parvapunctata KILENYI

Macrodentina (P.) proclivis Malz s. 1 .

Macrodentina (P.) steghausi (KLINGLER) s. 1 .

Macrodentina (M.) sp. 1 KILENYI

Mandelstamia (M.) rectilinea MALz

* Nodophthalmocythere tripartita MaLz

Orthonotacythere interrupta TRIEBEL (confined to the lower part of the zone)

Schuleridea (S.) triebeli (Steghaus) s. 1. (confined to the lower part of the zone)

The upper limit of the Zone is undefined due to the lack of ostracods in the type section - no doubt the result of the unsuitable lithologies for the extraction of microfossils - but certainly includes a part - if not the whole of the Eudoxus Zone.

\section{ZONE OF $M A N D E L S T A M I A$ (X.) $M A C U L A T A$ K I L E N Y I}

The paucity of species makes the definition of this zone rather tentative. The first appearence of the zonal index is taken as the lower limit whereas the upper limit of the Zone is based on the appearance of the succeeding $G$. spinosa fauna. Nevertheless the fauna is very distinct (species marked * are confined to the Zone):

* Mandelstamia (X.) maculata KILENYI

* Cytheropteron sp. KILENYI

* Eocytheropteron aquitanum (DONZE)

Schuleridea (S.) moderata n. sp.

\section{ZONE OF $G A L L I A E C Y T H E R I D E A S P I N O S A$}

\section{K I L E N Y I}

This Zone has again a very characteristic and easily recognizable fauna. The lower limit is taken at the first appearence of Mandelstamia (X.) tumida n. sp., Galliaecytheridea spinosa KILENYI and Orthonotacythere pustulata 
KILENYI, the upper limit is very sharp, being marked by the simultaneous disappearence of the whole faunal assemblage of the Zone. No corresponding break in lithology can be observed, the upper zonal limit falling within the Lingula shales.

The following species occur in the Zone (species marked * are confined to the Zone):

* Galliaecytheridea spinosa KILENYI

* Aaleniella (D.) gracilis nov. sp.

* Aaleniella (D.) inornata (KILENYI)

* Dicrorygma (O.) brotzeni BRUUn Christensen

* Indet. gen B. sp. 1 KILENYI

* Mandelstamia (X.) tumida nov. sp. (confined to the lower part of the zone)

* Monoceratina sp. 1 and sp. 2 KILENYI

* Orthonotacythere pustulata KILENYI

* Orthonotacythere sp. KILENYI

* Paracypris problematica KILENYI

* Paracypris sp. 1 KILENYI

* Protocythere sigmoidea Steghaus (confined to the upper part of the zone)

* Protocythere nealei KILENYI (confined to the upper part of the zone) Schuleridea (S.) moderata n. sp. (confined to the lower part of the zone)

\section{ZONE OF GALLIAECYTHERIDEA POLITA}

\section{K I L E N Y I}

The presumably topmost ostracod zone of the Kimmeridgian has a very limited fauna of two species only; Galliaecytheridea polita KILENYI and Macrodentina (M.) foveata MALz. Yet the assemblage is characteristic as neither of these species have been found in beds below or above.

The presence of Galliaecytheridea compressa nov. sp. in the Portland Sand and in the uppermost $10 \mathrm{ft}$. of the Kimmeridge Clay (cf. p. 43-44) shows that a local range zone of $G$. compressa may be extended down in the Kimmeridgian. 


\section{RECOGNITION OF THE KIMMERIDGIAN OSTRACOD ZONES IN OTHER AREAS}

The biostratigraphical subdivision of the type section of the Kimmeridgian in Dorset makes it possible to correlate other biostratigraphical units directly with the Kimmeridgian Stage. In the subsequent pages, the authors make only a rough outline of such a correlation from other areas in Northern and Western Europe to the Dorset area, as information of most of these areas is based on the literature. While problems as the demarcations of Lower and Upper Kimmeridgian, and the recognition of species from the Dorset zones in other areas are discussed, detailed correlations with the Kimmeridgian is not attempted in this paper. Such detailed correlations are not practicable, until direct correlations between the different areas permit a more or less precise correlation to the type Kimmeridgian of Dorset, but this is outside the scope of this work.

\section{N O R T H G E R A N B A S N}

Marine Kimmeridgian deposits occur near the Dutch border, and the area had direct marine connection to the Polish Basin. The North German Basin was limited towards the North by the Ringkøbing-Funen Height of Denmark. The deposits are exposed in several localities in Northwest Germany and have been studied on the basis of ostracod faunas by KLINGLER (1955) and Schmidt (1955). Many palaeontological papers about ostracods have been published on the basis of material from boreholes (KLINGler, Malz and Martin 1962, Malz 1957, 1958 a, b, Martin 1958, 1959, Schmidt 1954, Steghaus 1951, 1953, and Triebel 1954). Added to those are papers with biostratigraphical evaluations of the Kimmeridgian ostracod faunas of Northwest Germany (KLingler 1956, Bischoff and Wolburg 1963) and a paper dealing with ostracods from the Kimmeridgian of Northeast Germany (WIENHOLz 1968).

The Upper Jurassic in Northwest Germany is divided into Unterer, Mittlerer, and Oberer Malm, which have been subdivided in numbered biostratigraphical zones. The Kimmeridgian includes the whole of the Mittlerer Malm (Kimmeridge) and the lowermost part of Oberer Malm: 
Portlandian ...... Münder Mergel (Oberer Malm 5-3)

Upper Kimmeridgian $\left\{\begin{array}{l}\text { ? Eimbeckhäuser Plattenkalk (Oberer Malm 2) } \\ \text { Gigas - Schichten (Oberer Malm 1) }\end{array}\right.$

Lower Kimmeridgian $\left\{\begin{array}{l}\text { Ober-Kimmeridge (Mittlerer Malm 3) } \\ \text { Mittel-Kimmeridge (Mittlerer Malm 2) } \\ \text { Unter-Kimmeridge (Mittlerer Malm 1) }\end{array}\right.$

Oxfordian ...... Korallenoolith (Unterer Malm 6-4)

The following ostracod species from the different zones in the chronostratigraphical type section of Kimmeridgian have been found in the North German Basin:

\section{A. LOWER KIMMERIDGIAN}

The two ostracod zones of the Lower Kimmeridgian of Dorset are clearly recognizable in the North German Basin and they seem to be the equivalents of the Mittlerer Malm 1-2. The following species are common to both areas.

\section{Zone of Galliaecytheridea dissimilis OerTLI s. str.}

Occurrence of the indexfossil $G$. dissimilis Oertu in North-West Germany has only been reported by Glashoff (1964, p. 38), who found the species in the Mittlerer Korallenoolit and Unter-Kimmeridge. The other species from the Zone are:

Galliaecytheridea dissimilis Oertur $s .1$.

Amphicythere (A.) confundens OERTLI

Eocytheropteron (E.) decoratum (ScHMIDT)

Galliaecytheridea gracilis GLASHOFF

Galliaecytheridea postrotunda OERTLI

Orthonotacythere interrupta TRIEBEL

Protocythere rodewaldensis (KLINGLER)

Schuleridea (S.) triebeli (Steghaus) s. 1.

\section{Zone of Galliaecytheridea elongata KILENYI}

The index fossil $G$. elongata Kileny does not seem to occur in the North German Basin, but the following species are in common with the type section of Dorset:

Cytherelloidea weberi STEGHAUS

Cytherelloidea paraweberi OERTLI

Exophthalmocythere fuhrbergensis STEGHAUS 
Macrodentina (M.) maculata MALZ

Macrodentina (P.) steghausi (KLINGLER) s. 1 .

Orthonotacythere interrupta TRIEBEL

Schuleridea (S.) triebeli (Steghaus) s. 1.

\section{B. UPPER KIMMERIDGIAN}

Only two of the species of the Upper Kimmeridgian in Dorset occur in the North German Basin, and therefore it is not possible to make a direct correlation of ostracod zones between the two areas.

Protocythere sigmoidea Steghaus and Dicrorygma (O.) brotzeni BRUUn Christensen from the G. spinosa Zone occur in North Germany. The first in Mittleren Kimmeridge and older deposits. The latter in the lower part of Mittleren Kimmeridge, and in Münder Mergel.

\section{NORTH WEST POLISH B ASIN}

This area comprises the North West Polish Lowlands, the Northeastern Germany, and Scania in South Sweden. A number of biostratigraphical papers with lists of ostracod species have recently been published by Polish micropaleontologists (BIELECKA \& STYK 1963, 1964, 1968) which allow us to give a reasonably complete list of species from the Polish Lowlands. These publications are supplemented by papers by Oertli, Brotzen, and Bartenstein (1961), and BruUn Christensen (1965, 1968, various manuscripts) from Scania and Southeastern Denmark.

The deposits in this North West Polish Basin are mostly composed of clays and limestones, but in many parts they have not been investigated and described in detail. In the most northern part of the basin, in Scania, the Kimmeridgian deposits comprise the more or less sandy Fyledal Clay locally with thin beds of limestones (BRUUN CHRISTENSEN 1968) with sandy beds without ostracods below and above this formation.

The ostracod faunas from the basin show a marked biostratigraphic connection to the North German Basin throughout the Kimmeridgian and a somewhat less pronounced connection to the East to basins in the U.S.S.R. A connection to the North Western Danish Embayment through the DanishPolish Trough existed in the Lower Kimmeridgian, while a faunal barrier between the two basins came into being in the Upper Kimmeridgian. These relations appear by comparisons between the whole faunas in the basins as well as by the faunal development in the Fyledal Clay.

As in North Germany, the Polish and the Danish geologists employ the stratigraphical term Kimmeridgian for the Lower Kimmeridgian, while deposits of Upper Kimmeridgian Times have been classified as Portlandian (cf. Bruun Christensen 1968) or Bononian. 
A comparison of the ostracod faunas in the North West Polish Basin with the faunas from the type deposits of Kimmeridgian in Dorset shows that the following species are in common and are found in respectively Lower Kimmeridgian ( = dolny and gorny kimmeryd) and Upper Kimmeridgian ( $=$ svodkowy bonon):

\section{A. LOWER KIMMERIDGIAN}

\section{Zone of Galliaecytheridea dissimilis OERTLI s. str.}

This Zone seems to be recognizable by having following species in common with the Dorset area:

Galliaecytheridea dissimilis OeRTLI s. 1 .

Amphicythere (A.) confundens OERTLI

Eocytheropteron (E.) decoratum (SснмIDT)

Galliaecytheridea dorsetensis n. sp.

Galliaecytheridea postrotunda OERTLI

Macrodentina (P.) proclivis MaLz s. 1 .

Protocythere rodewaldensis (KLINGLER)

Orthonotacythere interrupta TRIEBEL

Schuleridea (S.) triebeli (Steghaus) s. 1.

\section{Zone of Galliaecytheridea elongata KILENYI}

The following species are in common with those of the Zone in Dorset:

Cytherelloidea paraweberi OERTLI

Exophthalmocythere fuhrbergensis STEGHAus

Galliaecytheridea dissimilis Oertui s. 1.

Macrodentina (P.) proclivis MaLz s. 1 .

Orthonotacythere interrupta TRIEBEL

Schuleridea (S.) triebeli (Steghaus) s. 1 .

\section{B. UPPER KIMMERIDGIAN}

The three ostracod zones of the Upper Kimmeridgian can not be recognized in the North West Polish Basin. Few species such as Dicrorygma (O.) brotzeni BruUn Christensen and Protocythere sigmoidea Steghaus are in common between the two areas.

\section{NORTH WEST DANISH EMBA YMENT}

The Danish Embayment is the northwestern part of the Danish-Polish Trough. The Jurassic and Lower Cretaceous ostracod faunas from this area have been studied by BRUUN CHRISTENSEN mainly on basis of material 
from boreholes. Very little has as yet been published about the faunas of Kimmeridgian Age. The area of Scania (Bruun Christensen 1968a, b) naturally belongs to the North West Polish Basin, while the Kimmeridgian deposits found in boreholes in Northern Jylland naturally belong to a North West Danish Embayment separated from the North West Polish Basin in the Upper Kimmeridgian by a faunal barrier.

The Kimmeridgian deposits of the North West Danish Embayment consists of a relatively thick series of sand and clay formations (fig. 3). Different ostracod faunas from brackish-marine deposits near the margin of the basin to marine ones in the middle of the basin have been recognized.

All the Kimmeridgian ostracod zones of Dorset with exception of one, have been found in the North West Danish Embayment, and elements of ostracod faunas from the type Portlandian can as well be recognized. The following species from the Zones of Dorset occur in the area:

\section{A. LOWER KIMMERIDGIAN}

\section{Zone of Galliaecytheridea dissimilis OERTLI s. str.}

Galliaecytheridea dissimilis OERTLI s. str.

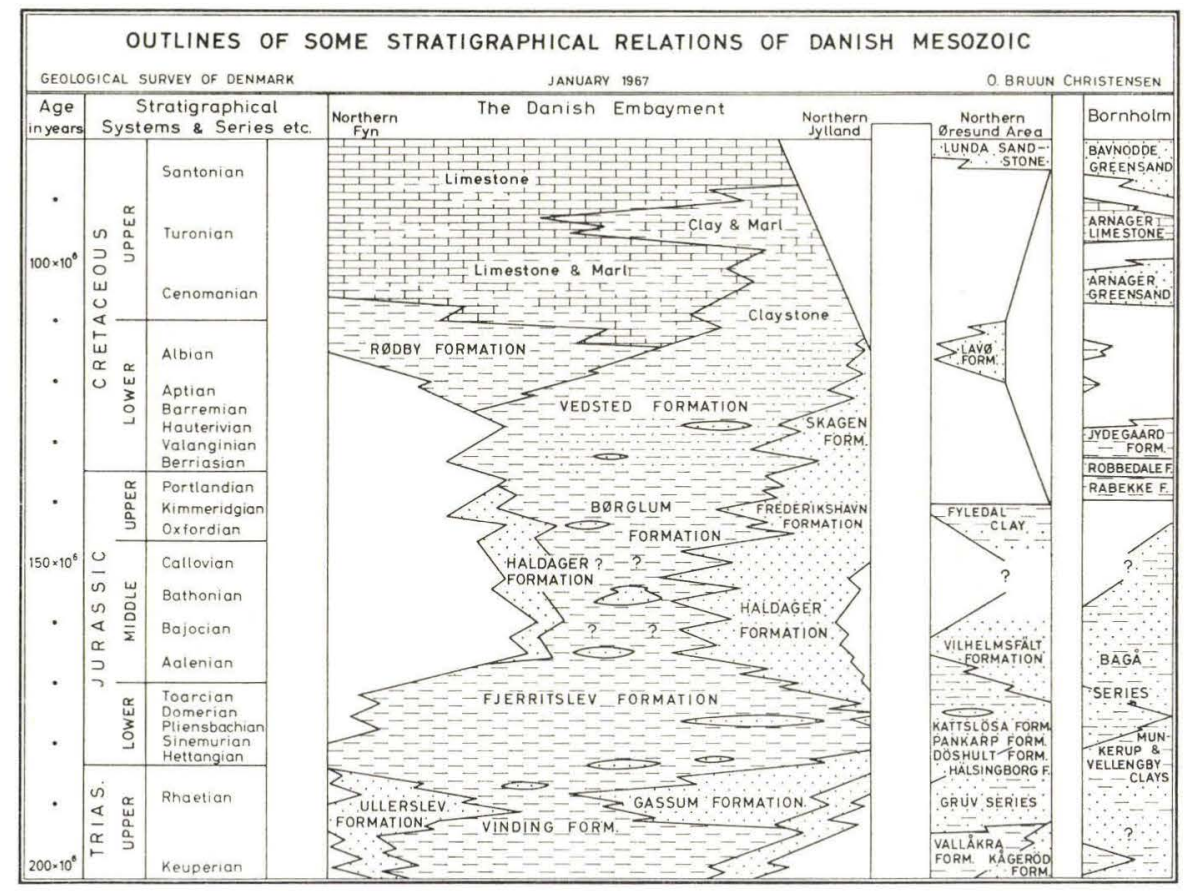

Fig. 3. An outline with some of the lithostratigraphical units of the northwestern and southeastern Danish Embayment and their geochronological and regional relations. Based largely on LARSEN (1966). 
Galliaecytheridea dorsetensis n. sp.

Galliaecytheridea gracilis GLASHOFF

Galliaecytheridea postrotunda OERTLI

Mandelstamia (M.) rectilinea MALz

Orthonotacythere interrupta TRIEBEL

? Protocythere rodewaldensis (KLINGLER)

Schuleridea triebeli (Steghaus) s. 1.

\section{Zone of Galliaecytheridea elongata KILENYI}

Galliaecytheridea elongata KILENYI

? Dicrorygma (O.) reticulata BRUUn Christensen (see p. 50)

Galliaecytheridea dissimilis OertLI s. 1 .

Galliaecytheridea dorsetensis n. sp.

Mandelstamia (M.) rectilinea MALZ

Orthonotacythere interrupta TRIEBEL

Schuleridea (S.) triebeli (Steghaus) s. 1.

B. UPPER KIMMERIDGIAN

\section{Zone of Mandelstamia (X.) maculata KILENYI}

This Zone does not seem to be recognizable in the Danish Embayment since Mandelstamia (X.) maculata KILENYI does not seem to occur in the area. Schuleridea (S.) moderata n. sp., which is found in this Zone in Dorset, occurs in the G. spinosa Zone in the Danish Embayment.

\section{Zone of Galliaecytheridea spinosa KILENYI}

Galliaecytheridea spinosa KILENYI

Aaleniella (D.) gracilis n. sp.

Aaleniella (D.) inornata (KILENYI)

Dicrorygma (O.) reticulata BRUUn CHRISTENSEN (see p. 50)

Mandelstamia (X.) tumida n. sp.

Orthonotacythere pustulata KILENYI

Protocythere sigmoidea STEgHaus

Schuleridea (S.) moderata n. sp.

\section{Zone of Galliaecytheridea polita KILENYI}

Few specimens of G. polita KILENy hav been found in the Danish Embayment, but the occurrence of Galliaecytheridea compressa nov. sp. and some other species indicate faunas of Lower Portlandian Age above the Kimmeridgian faunas. 


\section{P A R IS B A S I N}

The ostracod fauna of the Paris Basin and its vertical distribution is based largely on material described from the Vernon 1 borehole $(80 \mathrm{~km} \mathrm{NW}$ of Paris) by Oertli (1957) with additional data by Malz (1958) from three localities (E and W of Paris), Guyader (1962) (borehole in Le Havre) and OertLi (1963). The fauna is predominantly from the Lower Kimmeridgian.

\section{A. LOWER KIMMERIDGIAN}

\section{Zone of Galliaecytheridea dissimilis OeRTLI s. str.}

This zone is clearly recognizable in the Vernon borehole with its characteristic faunal assemblage. The lower boundary coincides precisely with the Kimmeridgian/Oxfordian boundary as OERTLI (1957) marks it on his distribution chart. We consider the upper limit of the zone to be in approximately the middle of OERTLI's "Kimmeridgien inferieur, partie moyen".

The characteristic assemblage consists of:

Galliaecytheridea dissimilis OERTLI $S$. str.

Amphicythere (A.) confundens (OERTLI)

Eocytheropteron (E.) decoratum (SCHMIDT)

Schuleridea (S.) triebeli (Steghaus) s. 1.

The whole fauna is very similar to that of the zone in Dorset but with some important differences. Absent from the Paris Basin is Macrodentina (M.) cicatricoda (the dominant species of the Zone in Dorset) and there is no trace of the genus Mandelstamia species of which are in common in Dorset.

\section{Zone of Galliaecytheridea elongata KILENYI}

This zone is also easily recognizable in the Vernon borehole. The base of it is marked by the association of Exophthalmocythere fuhrbergensis and Dicrorygma (O.) reticulata. These are joined by Orthonotacythere interrupta, Galliaecytheridea wolburgi s. 1., Schuleridea triebeli s. 1. The top of the profile is situated in this zone.

GUYADER (1962 p. 26) mentions an ostracod faunule from the Le Havre borehole (Zone des "calcaire coquilliers") which appears to belong to $G$. dissimilis and basal $G$. elongata Zones.

\section{B. UPPER KIMMERIDGIAN}

The close similarity which exists between the Lower Kimmeridgian faunas of the Paris Basin and Dorset is reduced to practically nil in the Upper 
Kimmeridgian. Only one species, Eocytheropteron (E.) aquitanum is common to both localities (Boulogne sur le Mer, Oertli, 1963) suggesting the probable presence of the Mandelstamia (X.) maculata Zone here.

\section{SOUTH WEST FRANCE}

Upper Kimmeridgian ostracods were described from this area by MALZ (1958) and DONZE (1960). The fauna is totally different from that of the Upper Kimmeridgian of Dorset and no correlation is possible although Eocytheropteron (E.) decoratum occurs in both localities.

\section{SWISS J URA}

OertLi (1959) described an extensive Upper Jurassic fauna from a large number of localities in the Swiss Jura. The stratigraphical range of his samples spreads from the Lower Oxfordian to the Upper Kimmeridgian, although only two samples cover the basal part of the latter. In spite of the fact that seven species are common to the Lower Kimmeridgian of Dorset and the Swiss Jura, very great differences exists between the two areas. The most important is the complete absence of Galliaecytheridea here, a genus stratigraphically so important in all the other localities. Only very tentative correlations can therefore be made.

The Galliaecytheridea dissimilis s. str. Zone is indicated by a fauna which includes Amphicythere (A.) confundens, Protocythere rodewaldensis, Eocytheropteron (E.) decoratum, Cytherelloidea weberi and Schuleridea (S.) triebel $s$. $l$. The base of the zone coincides with the Oxfordian/Kimmeridgian boundary as shown by OerTLI (1959 text fig. 3). The Galliaecytheridea elongata Zone cannot be recognized. 


\section{ALPHABETICAL LIST OF OSTRACOD SPECIES IN THE KIMMERIDGIAN OF NORTHERN AND WESTERN EUROPE}

The following list is based on publications dealing with the ostracod faunas of the areas dealt with in this paper. In addition five species which are described for the first time are included.

The name of each species in the list is followed by its recorded presence in the areas considered, the letter $\mathrm{L}$ representing an occurrence in the Lower and $U$ in the Upper Kimmeridgian, according to the authors' correlations with the type section. The column on the extreme right gives the most recent reference to the species or the page number in this paper where it is discussed in detail. Only those species are annotated this way which are stratigraphically important or have a complex synonymy.

The list does not give the full complement of species mentioned from Northern and Western Europe as a number of taxa with dubious identities had to be excluded. For example some species in the Polish literature are mentioned by the name of the genus followed by the designation "sp. sp." and these have not been included. On the other hand species from other areas treated with open nomenclature are incorporated as separate identity for these can be assumed without much doubt. The exclusion of a certain number of the Polish species from the list is to a certain extent counterbalanced by the inclusion of all the species from Scania.

Galliaecytheridea compressa n. sp., which occurs in the Portland Sand of Dorset and the Børglum Formation of the Danish Embayment, has been reported by BARKER (1966) from the topmost part of the Kimmeridge Clay of Dorset. As the present authors have not found the species there it has not been included into the following list.

The list shows 201 species of ostracods in the Kimmeridgian of Northern and Western Europe. There are 98 species in deposits of Upper Kimmeridgian age, and 139 in those of Lower Kimmeridgian in age. 36 species are common to both. 
A LPHABETICAL LIST OF OSTRACOD SPECIES IN THE KIMMERIDGIAN OF NORTHERN AND WESTERN EUROPE

\begin{tabular}{|c|c|c|c|c|c|c|c|c|}
\hline \multirow[b]{2}{*}{$=$} & \multicolumn{8}{|c|}{ Areas with Kimmeridgian deposits } \\
\hline & $\begin{array}{l}\vec{\Delta} \\
\stackrel{0}{0} \\
\stackrel{0}{0} \\
\dot{-}\end{array}$ & 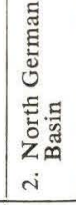 & 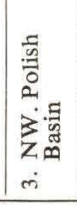 & 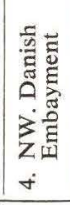 & 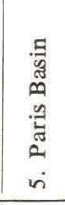 & 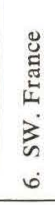 & $\begin{array}{l}\frac{\pi}{5} \\
\qquad \\
n \\
n \\
\vdots \\
n \\
r\end{array}$ & \\
\hline Aaleniella (Danocythere) gracilis nov. subgen. et sp. & $\mathrm{U}$ & & & $\mathrm{U}$ & . & & 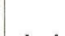 & see page 54 \\
\hline Aaleniella (Danocythere) inornata (KILENYI 1969) & $\mathrm{U}$ & . & . & $\mathrm{U}$ & . . & . & . & see page 56 \\
\hline Aaleniella (Danocythere) nov. sp. BRUUN CHRISTENSEN (MS) & . . & . & U & . & . & . & . & \\
\hline Acanthocythere (Unodentina) undata Malz 1958 & . . & . & . & . & $\mathrm{L}$. & . & . & \\
\hline Amphicythere (Amphicythere) confundens (OERTLI 1957) & L. & $\dot{\mathrm{L}}$. & $\mathrm{L}$ & . & $\mathrm{L}$. & . & L. & \\
\hline Amphicythere (Amphicythere) pennyi KILENYI 1969 & $\mathrm{~L}$. & . & . & . & . & . & . & \\
\hline Amphicythere (Merocythere) plena (Schmint 1954) s. $l$. & . . & $\mathrm{L}$. & . & $\mathrm{L}$. & $\mathrm{L}$. & $\mathrm{U}$ & . & \\
\hline Amphicythere (Amphicythere) semisulcata TrIeBeL 1954 & . . & L. & $\mathrm{L}$. & $\mathrm{L}$. & . . & . . & . . & see Klingler, Malz \& Martin 1962 \\
\hline Amphicythere (Amphicythere) sphaerulata KILENYI 1969 & L. & . & . . & . & . & . & . & \\
\hline Bairdia sp. BruUn Christensen (MS) & . . & . . & . . & U & . . & . . & . . & \\
\hline Bairdia? sp. KLINGLER 1955 & . . & $\mathrm{L}$. & . . & . . & . . & . . & . . & \\
\hline Bairdia sp. BruUn Christensen (MS) & . . & . . & U & . . & . . & . . & . . & \\
\hline Bythocypris sp. A OERTLI 1957 & . . & . & . . & . & $\mathrm{L}$. & . . & . . & \\
\hline Cetacella armata MARTIN 1958 & . . & L. & . . & . . & . . & . . & . . & \\
\hline Cetacella inermis MARTIN 1958 & . . & $\mathrm{L}$. & . . & . . & . . & . . & . . & see Klingler, Malz \& Martin 1962 \\
\hline Cetacella sp. BruUn Christensen (MS) & . . & . & U & $\mathrm{L}$. & . . & . & . & \\
\hline Cypridea sp. A DONZE 1960 & . . & . . & . . & . . & . . & . U & . . & \\
\hline Cypridea sp. B DONZE 1960 & . . & . & . . & . & . . & U & . & \\
\hline Cypridea sp. BRUUn CHRISTENSEN (MS) & . & . & $\mathrm{U}$ & . & . & . & . & \\
\hline "Cyprideis" sp. Donze 1960 & . & . & . & . & . & . U & . . & \\
\hline "Cyprideis"? derooi DONZE 1960 & . & . & . & . & . & . U & . & \\
\hline Cyprinotus? sp. Steghaus 1951 & . . & $\mathrm{L}$. & . . & . . & . . & . . & . . & \\
\hline Cythereis sp. SCHMIDT 1955 & . . & $\mathrm{U}$ & . . & . . & . . & . & . . & \\
\hline Cytherella recta SHARAPOVA 1939 & $\mathrm{~L}$. & . & . & . & . & . . & . . & $\begin{array}{r}\text { see KILENyI } 1969 \text { (Not C. recta Jones, } \\
\text { KIRBY \& Brady, 1884) }\end{array}$ \\
\hline
\end{tabular}




\begin{tabular}{|c|c|c|c|c|c|c|c|c|}
\hline & \multicolumn{8}{|c|}{ Areas with Kimmeridgian deposits } \\
\hline & 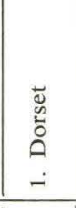 & 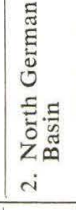 & 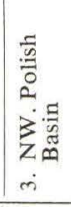 & 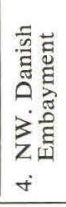 & 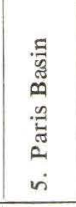 & 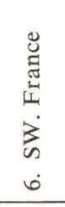 & 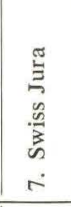 & \\
\hline Cytherella suprajurassica OERTLI 1957 & . . & L. & L. & . . & L. & . U &. & see Klngler, Malz \& Martin 1962 \\
\hline Cytherella sp. BRUUn CHRISTENSEN (MS) & . . & . . & $\mathrm{L}$. & . . & . . & . . & . . & \\
\hline Cytherelloidea paraweberi OERTLI 1957 & L. & $\mathrm{L}$. & L. & . . & L. & . . & L. & see page 40 \\
\hline Cytherelloidea undulata KLINGLER 1955 & . . & $\mathrm{L}$. & $\mathrm{L}$. & . & . & . . & . . & see Klingler,Malz \& Martin 1962 \\
\hline Cytherelloidea varicosa DonzE 1960 & . . & . . & . . & . . & . . & U & . . & \\
\hline Cytherelloidea weberi Steghaus 1951 & L. & L. & . . & . . & L. & . U & L. & See KilEnyt 1969 \\
\hline Cytherelloidea sp. A. OERTLI 1957 & . . & $\mathrm{L}$. & . . & . . & L. & . . & $\mathrm{L}$. & \\
\hline Cytherelloidea sp. SснміDт 1955 & . . & L. & . . & . . & . . & . . & . . & \\
\hline Cytheropteron acutissimum MARTIN 1957 & . & $\mathrm{L}$. & . . & . . & . . & . . & . . & \\
\hline Cytheropteron (Cytheropteron) bispinosum Sснм. 1954 s. l. & . & L U & L U & . . & $\mathrm{L}$. & . . & . . & \\
\hline Cytheropteron cf. prolongatum SHARAPOVA & . . & . U & . . & . . & . & . . & . . & see SснміDт 1955 \\
\hline Cytheropteron (Cytheropteron) purum ScнміDт 1954 s. $l$. & . . & L U & L U & . . & . U & . . & . . & \\
\hline Cytheropteron? n. sp. Donze 1960 & . . & . . & . . & . . & . . & U & . . & \\
\hline Cytheropteron? sp. KILENYI 1969 & . U & . . & . . & . . & . . & . . & . . & \\
\hline Cytheropteron? nov. sp. BRUUN CHRISTENSEN (MS) & . . & . . & . U & . . & . . & . . & . . & \\
\hline Cytherura? sp. A. Oerthi 1957 & . . & . . & . . & . . & L . & . . & . . & \\
\hline Cytherura? B. OERTLI 1957 & . . & . . & & . . & L . & . . & . . & \\
\hline Darwinula leguminella FORBES 1855 & . . & L U & . & . . & . . & . & . . & see Glashoff 1964 \\
\hline Darwinula oblonga (RoEMER 1839) & . . & $\mathrm{L}$. & . . & . & . & . . & . . & see Glashoff 1964 \\
\hline Darwinula sp. DonZe 1960 & . . & . . & . . & . . & . . & . U & . . & \\
\hline Dicrorygma (Orthorygma) brotzeni BRUUN CHRISTENSEN 1965 &. $\mathrm{U}$ & L U & $\mathrm{L} \mathrm{U}$ & & . . & . . & . . & see page 50 \\
\hline Dicrorygma (Orthorygma) reticulata BRUUN CHRISTENSEN 1965 & L. & . . & . . & . U & $\mathrm{L}$ & & . . & see page 49 \\
\hline Dicrorygma (Orthorygma) major BRUUN CHRISTENSEN 1965 & . . & . . & . . & . U & . . & . . & . . & \\
\hline Eocytheropteron aquitanum (DONZE 1960) & . U & . . & . . & . . & . U & . U & . . & See Kilenyi 1969 \\
\hline Eocytheropteron decoratum (ScнмIDT 1954) s. $l$. & L. & L U & L U & . . & $\mathrm{L}$. & . . & L U & \\
\hline Eucypris? sp. MARTIN 1940 & . . & . U & . . & . . & . . & . . & . . & see SCHMIDT 1955 \\
\hline Eucythere? sp. BRUUn CHRISTENSEN (MS) & . . & . . & $\mathrm{L}$. & . . & . . & . . & . . & \\
\hline
\end{tabular}


Eucytherura n. sp. BRUUn CHRISTENSEN (MS)

Eucytherura (Vesticytherura) n. sp. BRUUN CHR. (MS) Exophthalmocythere gigantea ScHMIDT 1954

Exophthalmocythere fuhrbergensis STEGHAUs 1951

Fabanella prima MARTIN 1961

Fabanella aff. polita ornata STEGHAUs 1951

Fabanella nov. sp. BRUUn CHRISTENSEN (MS)

Galliaecytheridea confundens KILENYI 1969

Galliaecytheridea dissimilis OeRTLI 1957 s. $l$.

Galliaecytheridea dorsetensis nov. sp.

Galliaecytheridea elongata KILENYI 1969

Galliaecytheridea fragilis KILENYI 1969

Galliaecytheridea gracilis GLASHOFF 1964

Galliaecytheridea? hiltermanni (STEGHAUS)

Galliaecytheridea? mandelstami (LuUBIMOVA 1955)

Galliaecytheridea $\mathrm{cf}$. mandelstami (Lubimova 1955)

Galliaecytheridea minuta (ScHмIDT 1955)

Galliaecytheridea? monstrata (LJuBIMOVA 1955)

Galliaecytheridea oertlii nov. sp.

Galliaecytheridea? pfannenstieli (STEGHAUs 1951)

Galliaecytheridea polita KILENYI 1969

Galliaecytheridea postrotunda OERTLI 1957

Galliaecytheridea spinosa KILENYI 1969 s. $l$.

Galliaecytheridea wolburgi (STEGHaUs 1951)

Galliaecytheridea sp. 1 KILENYI 1969

Galliaecytheridea? nov. sp. BRUUN CHRISTENSEN (MS)

Galliaecytheridea nov. sp. BruUn CHRISTENSEN (MS)

Galliaecytheridea nov. sp. BRUUN CHRISTENSEN (MS)

Galliaecytheridea nov. sp. BRUUN CHRISTENSEN (MS)

Galliaecytheridea nov. sp. BRUUN CHRISTENSEN (MS)

Galliaecytheridea nov. sp. BRUUN CHRISTENSEN (MS)

Galliaecytheridea nov. sp. BRUUN CHRISTENSEN (MS)

Galliaecytheridea nov. sp. BRUUN CHRISTENSEN (MS)

Galliaecytheridea sp. BruUn CHRISTENSEN (MS)

Galliaecytheridea sp. BRUUn CHRISTENSEN (MS)

Galliaecytheridea sp. BruUn CHRISTENSEN (MS)

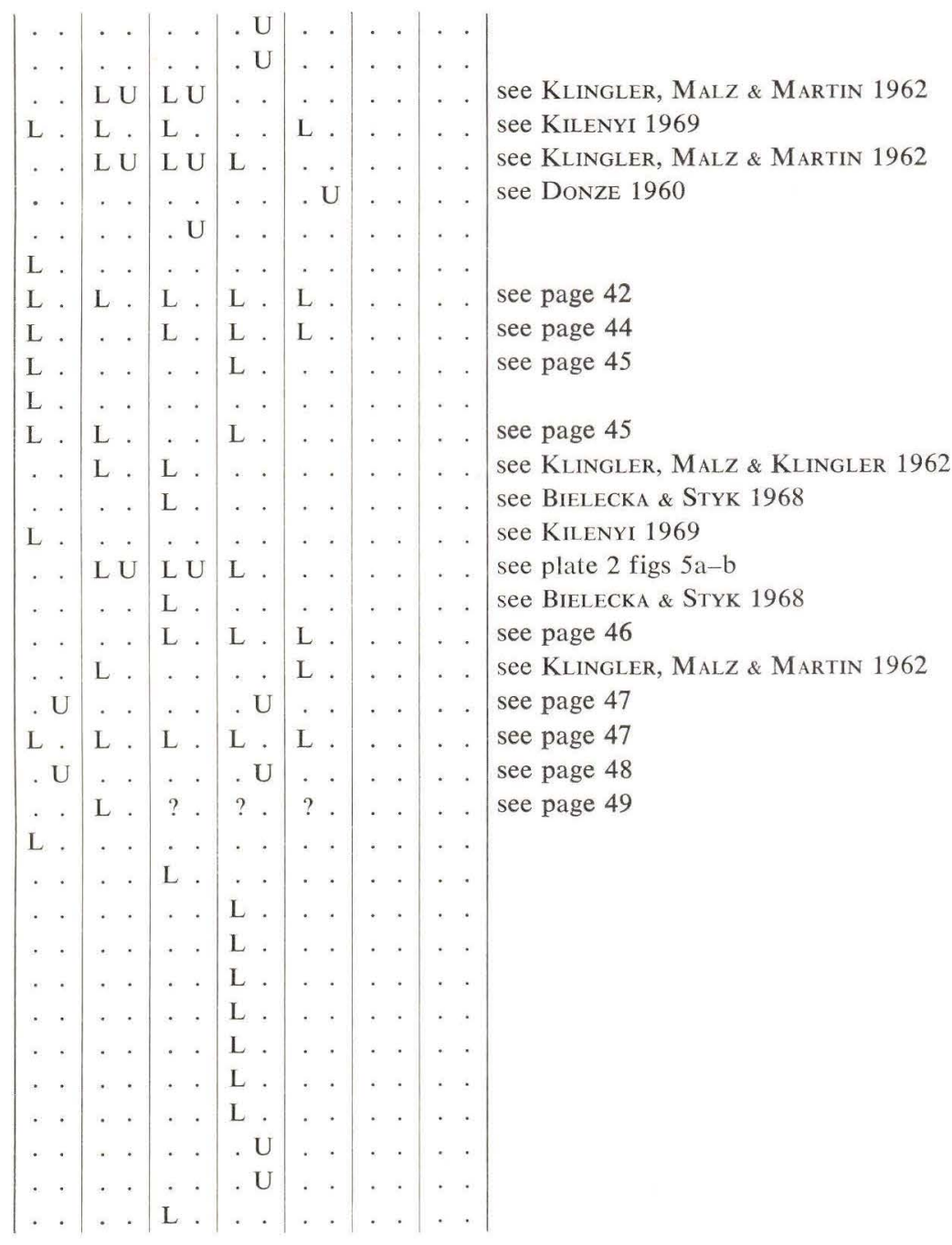




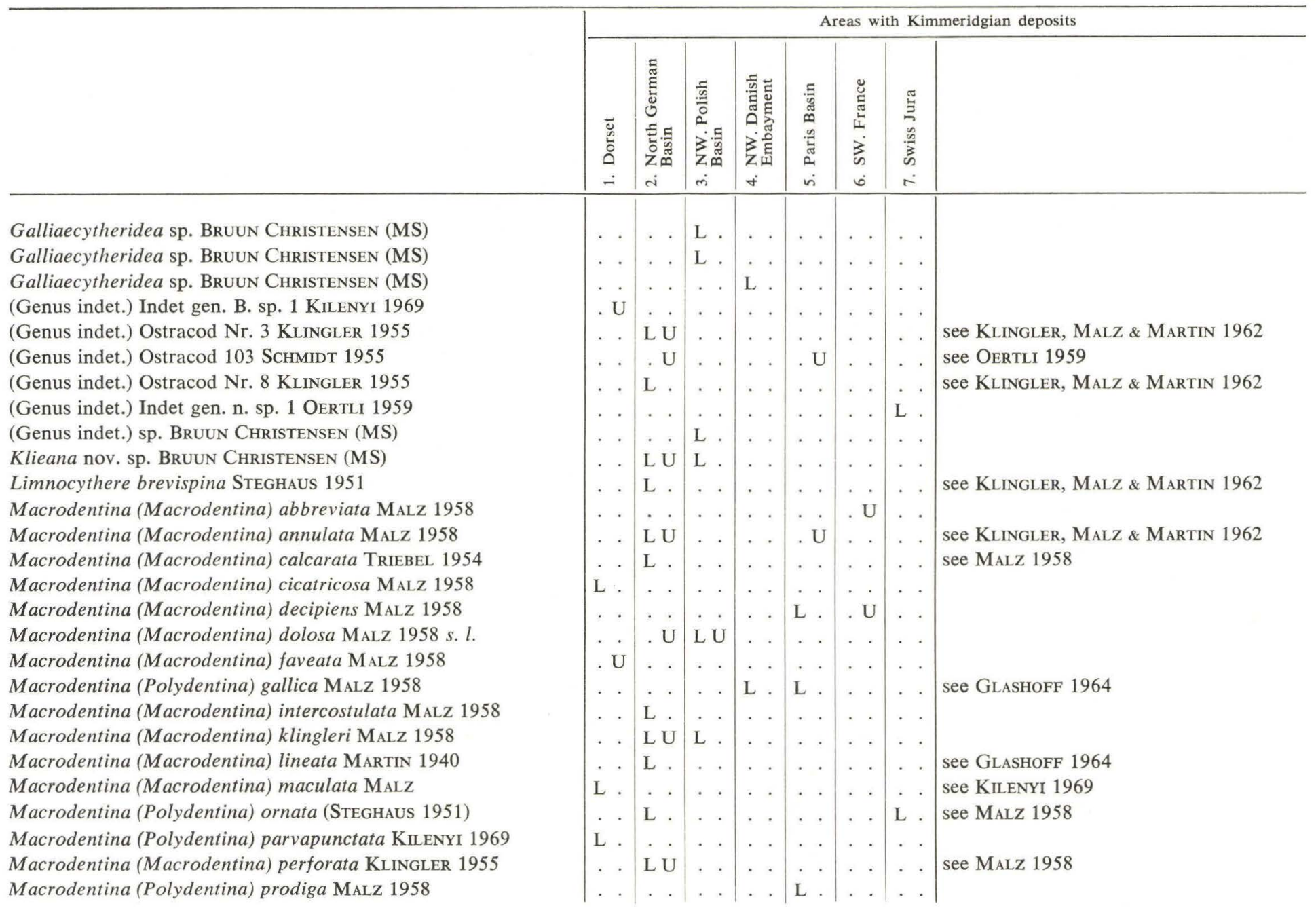


Macrodentina (Polydentina) proclivis MALz 1958 s.l. Macrodentina (Polydentina) pulchra (SchmidT 1955) Macrodentina (Macrodentina) punctata OERTLI 1957 Macrodentina (Macrodentina) punctatula MALZ 1958 Macrodentina (Polydentina) ramulosa MALz 1958

Macrodentina (Polydentina) rudis MALz 1958 s. $l$. Macrodentina (Macrodentina) sculpta KLINGLER 1955 Macrodentina (Polydentina) steghausi (KLINGLer 1955) s. $l$. Macrodentina (Polydentina) wicheri (Steghaus 1951) s. $l$.

Macrodentina (Polydentina) nov. sp. BruUn CHr. (MS) s. $l$. Macrodentina sp. BRUUN Christensen (MS)

Macrodentina sp. BRUUN CHRISTENSEN (MS)

Mandelstamia (Mandelstamia) angulata KILENYI 1969 Mandelstamia inflata (STEGHAUs 1951) $s$. I.

Mandelstamia (Xeromandelstamia) maculata KILENYI 1961 Mandelstamia (Mandelstamia) rectilinea MALZ 1958

Mandelstamia (Xeromandelstamia) tumida nov. sp. Mandelstamia sexti NeALE 1961 s. $l$.

Mandelstamia nov. sp. BRUUN CHRISTENSEN (MS)

Mandelstamia nov. sp. BRUUN CHRISTENSEN (MS)

Mandelstamia sp. BRUUn CHRISTENSEN (MS)

Mandelstamia sp. BRUUn CHRISTENSEN (MS)

Metacytheropteron elegans OERTLI 1957

Monoceratina saxonica SCHMIDT 1954

Monoceratina scrobiculata TRIEBEL \& BARTENSTEIN 1938

Monoceratina sp. 1 KILENYI 1969

Monoceratina sp. 2 KILENYI 1969

Monoceratina sp. BRUUN CHRISTENSEN (MS)

Monoceratina sp. BRUUN CHRISTENSEN (MS)

Monoceratina sp. BRUUN CHRISTENSEN (MS)

Nodophthalmocythere tripartita MALZ 1958

Nodophthalmocythere vallata MALZ 1958

Orthonotacythere cf. favulata MARTIN 1940

Orthonotacythere interrupta TRIEBEL 1941

Orthonotacythere kostytschevkaensis LuUBIMOVA

Orthonotacythere pustulata KILENYI 1969

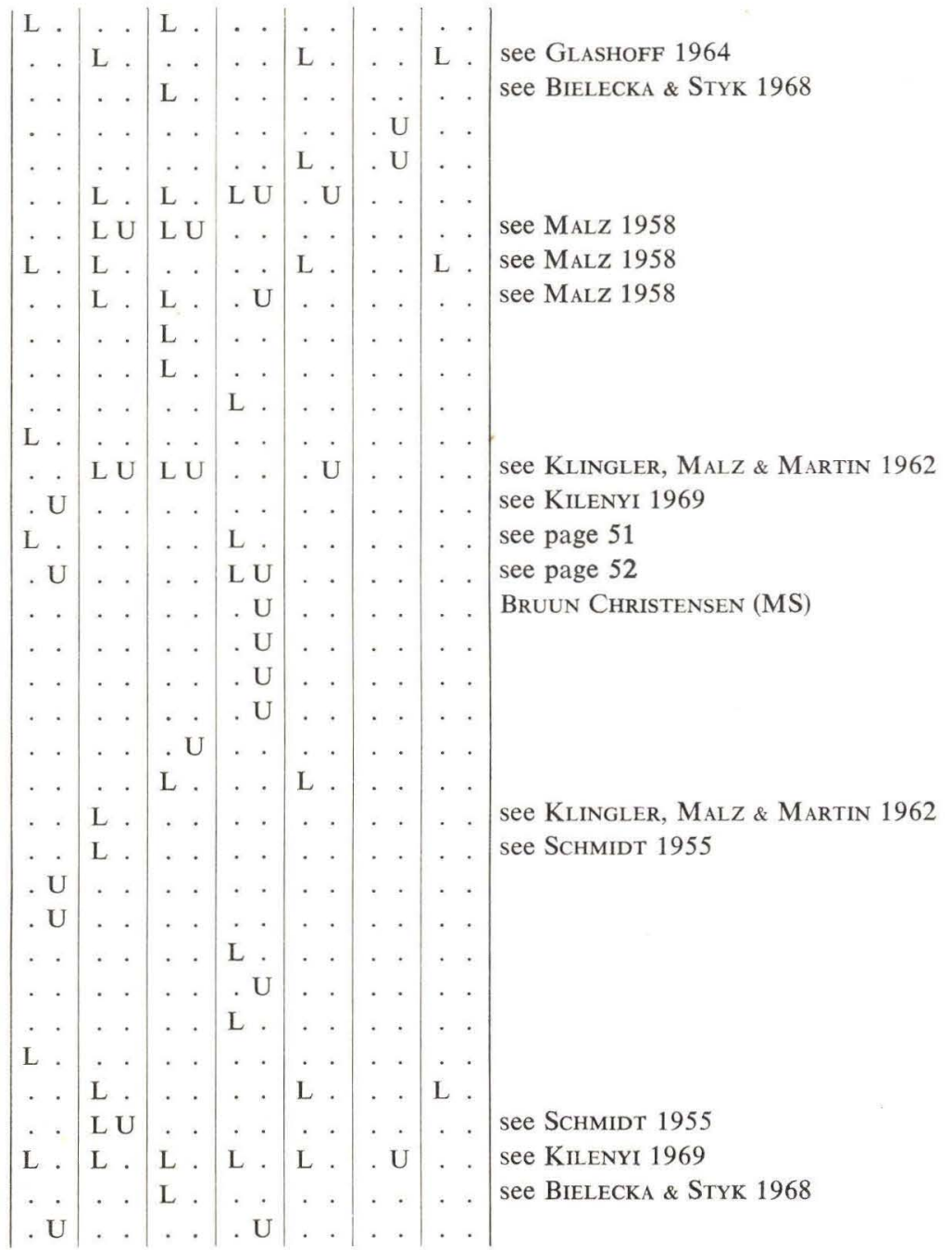




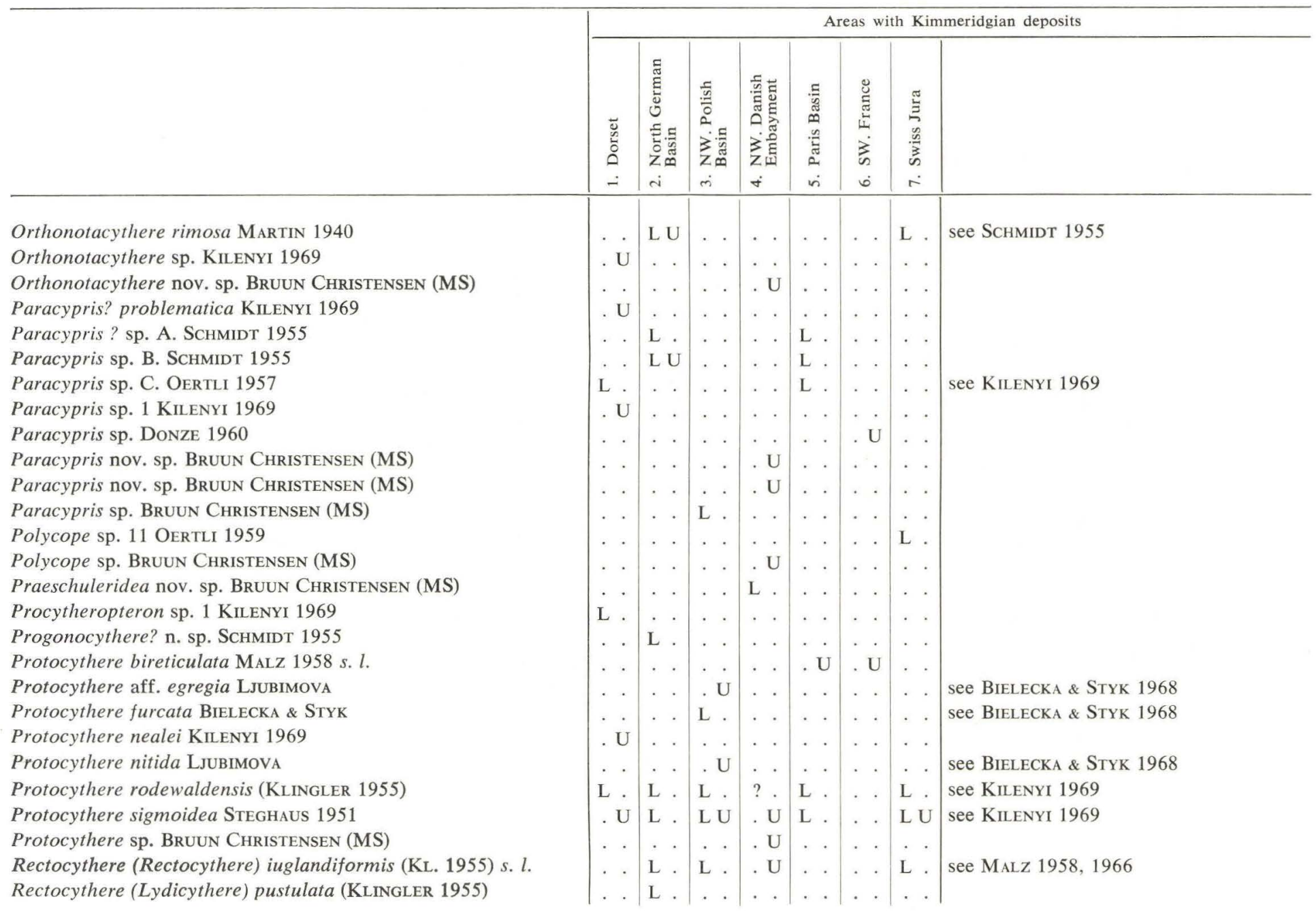


Rectocythere (Rectocythere) regularis MALZ 1958

Rectocythere (Rectocythere) rugosa MaLz 1966

Rectocythere (Lydicythere) scania nov. sp.

Rectocythere (Lydicythere) nov. sp. BRUUN CHRISTENSEN (MS)

Rectocythere (Lydicythere) sp. 1 (KILENYI 1969)

Rhinocypris jurassica (MARTIN 1940) s. l.

Scabriculocypris goerlichi KLINGLER 1955

Schuleridea (Schuleridea) moderata nov. sp.

Schuleridea? suprajurassica Donze 1960

Schuleridea (Schuleridea) triebeli (Steghaus 1951) s. $l$.

Schuleridea nov. sp. OeRTLi 1959

Schuleridea? sp. BRUUn CHRISTENSEN (MS)

Schuleridea? nov. sp. BRUUN CHRISTENSEN (MS)

Schuleridea (Eoschuleridea) nov. sp. BRUUN CHRISTENSEN (MS)

Schuleridea (Eoschuleridea)? sp. BRUUN CHRISTENSEN (MS

Schuleridea sp. BruUn CHRISTENSEN (MS)

Schuleridea sp. BRUUn CHRISTENSEN (MS)

Schuleridea sp. BRUUn CHRISTENSEN (MS)

Schuleridea nov. sp. BRUUn CHRISTENSEN (MS)

Theriosynoecum planiverrucosa (KLINGLER 1955)

Theriosynoecum? sp. (KLINGLER 1955)

Theriosynoecum sp. (SснміDт 1955)

Theriosynoecum sp. BRUUN CHRISTENSEN (MS)

Vernoniella sequana OERTLI 1957

\begin{tabular}{|c|c|c|c|c|c|c|c|}
\hline . . & . . & $\mathrm{L}$. & . . & L . & . . & $\cdot \cdot$ & \\
\hline . . & . . & . . & . . & . . & . U & . . & \\
\hline . . & . . & . . & $\mathrm{L}$. & . . & . . & . . & \\
\hline . . & . . & L . & L . & . . & . . & . . & see page 58 \\
\hline $\mathrm{L}$. & . $\cdot$ & . $\cdot$ & . . & . . & . . & . . & \\
\hline . . & $\mathrm{L} \mathrm{U}$ & $\mathrm{L}$. & . . & . . & . U & . . & see Glashoff 1964 \\
\hline . . & $\mathrm{L} \mathrm{U}$ & $\mathrm{L}$. & . . & . . & . . & . . & see Klingler, MaLZ \& Martin 1962 \\
\hline $\mathrm{U}$ & .. & . $\cdot$ & . U & . . & . . & . . & see page 41 \\
\hline . . & . . & . . & . . & . . & . $\mathrm{U}$ & . . & \\
\hline L . & L U & $\mathrm{L} \mathrm{U}$ & L ? & $\mathrm{L} \mathrm{U}$ & U & $\mathrm{LU}$ & see Glashoff 1964 \\
\hline . . & . . & . . & . . & . . & . . & $\mathrm{L}$. & \\
\hline . . & . .. & . U & $\mathrm{L}$. & . . & . . & . . & \\
\hline . . & . . & . . & L . & . . & . . & . . & \\
\hline .. & .. & $\mathrm{L}$. & . . & . . & . . & . . & \\
\hline . . & . . & . U & . . & . . & . . & . . & \\
\hline$\cdot \cdot$ & $\cdot \cdot$ & $\cdot \cdot$ & $\mathrm{L}$. & . . & . . & $\cdot \cdot$ & \\
\hline . . & . . & . . & L . & . . & . . & . . & \\
\hline$\cdot \cdot$ & $\cdot \cdot$ & $\cdot \cdot$ & $\mathrm{L}$. & . . & . . & . $\cdot$ & \\
\hline . . & . $\cdot$ & . $\cdot$ & L U & . . & . $\cdot$ & . $\cdot$ & \\
\hline . . & $\mathrm{L}$. & . . & . . & . . & . . & . . & see KLINGLER, Malz \& Martin 1962 \\
\hline$\cdot \cdot$ & $\mathrm{L}$. & . $\cdot$ & . . & . . & . . & . . & see Klingler, Malz \& Martn 1962 \\
\hline . . & $\mathrm{L}$. & . . & . . & . . & . . & . . & \\
\hline . $\cdot$ & . $\cdot$ & . U & . . & . . & . $\cdot$ & . $\cdot$ & \\
\hline . & . . & . . & . . & L. & . . & . . & \\
\hline
\end{tabular}




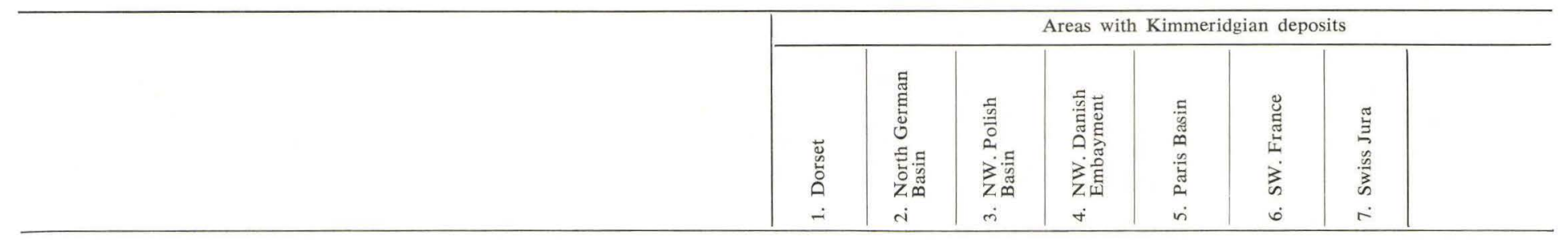

Number of species in the local areas.

(in brackets percentage of the total number of species)

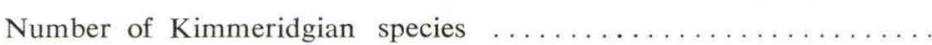

Number of Upper Kimmeridgian species ..................

Number of Lower Kimmeridgian species $\ldots . \ldots \ldots \ldots \ldots \ldots$.

$\begin{array}{llllllll}52(26) & 71(35) & 65(32) & 65(32) & 45(22) & 22(11) & 17(8) & \text { Total } 20\end{array}$

$\begin{array}{llllllll}20(20) & 26(27) & 24(24) & 30(31) & 9(9) & 22(22) & 3(3) & \text { Total } 98\end{array}$

Number of species occasionally occuring in Upper and Lower

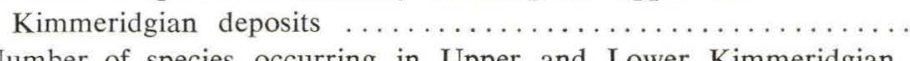

Number of species occurring in Upper and Lower Kimmeridgian

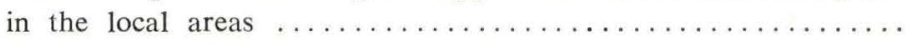

$\begin{array}{llllllll}7(19) & 30(83) & 23(63) & 13(36) & 16(36) & 7(19) & 6(17) & \text { Total } 36\end{array}$

$\begin{array}{lllllll}0 & 21 & 12 & 3 & 1 & 0 & 3\end{array}$

Number of species common between the areas.

Numbers of species in common (C)

between different areas ( $\mathrm{A}$ and $\mathrm{B}$ ).

In brackets percentage-species

(Jaccard Coefficient):

$$
\left(\frac{C}{A+B-C} \%\right)
$$

\begin{tabular}{|c|c|c|c|}
\hline Area & 1 & & \\
\hline - & 2 & & \\
\hline - & 3 & & $\mathrm{M}$ \\
\hline - & 4 & & $\mathrm{M}$ \\
\hline & 5 & & $\mathrm{~K}$ \\
\hline - & 6 & L & $D$ \\
\hline - & 7 & $\mathrm{O}$ & $G$ \\
\hline & & W & \\
\hline & & $\mathrm{E}$ & \\
\hline & & $\mathrm{R}$ & 1 \\
\hline
\end{tabular}

U K

$\mathrm{P}$ I

\begin{tabular}{lllllll}
$1(2)$ & $2(5)$ & $8(19)$ & $1(4)$ & $1(2)$ & $1(5)$ & P \\
\hline
\end{tabular}

$12(14) \backslash \begin{array}{llllll}11(28) & 1(2) & 5(17) & 2(4) & 2(7) & \mathrm{E} \mathrm{M} \\ \mathrm{R} & \mathrm{E} & -2\end{array}$

$11(14) 31(35) \backslash 2(4) \quad 3(10)$ 1( 2) $3(13) \quad \mathrm{R}-3$

$9(18) \quad 12(13) \quad 13(17) \backslash \quad 2(5) \quad 1(2) \quad 2(6) \quad I \quad-4$

$14(25) \quad 22(27) \quad 17(23) 10(14) \backslash 3(11) \quad 1(9) \quad G-5$

$\begin{array}{lllllll}0 & 0 & 0 & 0 & 0 & \backslash & 1(4) \\ \mathrm{I} & -6\end{array}$

$\begin{array}{llllll}7(17) & 14(20) & 7(11) & 2(4) & 11(26) & 0\end{array} \quad \mathrm{~N}-7$ 


\section{STRATIGRAPHICAL AND REGIONAL CONCLUSIONS}

In this work the authors have given only the species and their presence or absence in those deposits which in the basin mentioned have been classified as either Upper or Lower Kimmeridgian.

A study of the composition of the faunas with a compilation of ostracod assemblages and correlation of these between the seven areas of deposition will have great stratigraphical and palaeoecological importance. In the present work, only the composition of the faunas in the particular areas of deposition in Northern and Western Europe from the entire Upper and Lower Kimmeridgian will be set in relation to the type profiles for the Upper and Lower Kimmeridgian in Dorset and the stratigraphical distribution of the species which have been found in the type section will be examined in the various areas.

Figs. 4-7 (p. 36) show the number of species occurring in the various areas of deposition and the extent to which these areas have species in common. In the Lower Kimmeridgian (fig. 5 p. 36) relatively many species are common to these areas. The largest percentages of species in common are found between the North German Basin, the Paris Basin, and the North West Polish Basin. In the area of South West France no Lower Kimmeridgian ostracods have been found. In the Upper Kimmeridgian (fig. 7, p. 37) the distribution of species shows that a clear Southwest-Northeast trending faunal barrier developed, separating an Upper Jurassic North Sea Basin, comprising the Dorset area and the North West Danish Embayment of subsidence, from a Mid-European Region including the North German Basin, the North West Polish Basin, and the Paris Basin. At the same geological time the faunal similarity with the areas of South-Western France and the area in Switzerland is somewhat less developed. The area of SouthWestern France differs from the others in having more or less limnic environments. In the Swiss Jura in the Upper-Kimmeridgian deposits there are species of ostracods, and these have all been found also in deposits classified as Lower Kimmeridgian in other areas.

The faunal barrier between the North Sea Basin and the Mid-European Region, which became effective in the Upper Kimmeridgian, may be shown to be present also in the Portlandian. This barrier appears more clearly

3* 


\section{LEGEND TO FIGS. 5-7}

A Areas, with total number of species.

(0) In brackets, number of species occur-

(8) ring both in Lower and Upper Kimme-

(0) ridgian in the particular area.

$\bar{\equiv} \mathrm{C} \equiv$ Number of species in common.

Percentage species: $\frac{C}{A+B-C} \%$

$$
\text { (Example: } \frac{8}{20+30-8} \%=19 \% \text { ) }
$$

$--1-5 \%$

$\overline{\underline{ }}==6-10 \%$

$\overline{\overline{\bar{e}}} \equiv \equiv \equiv 11-15 \%$

etc.

Fig. 4.

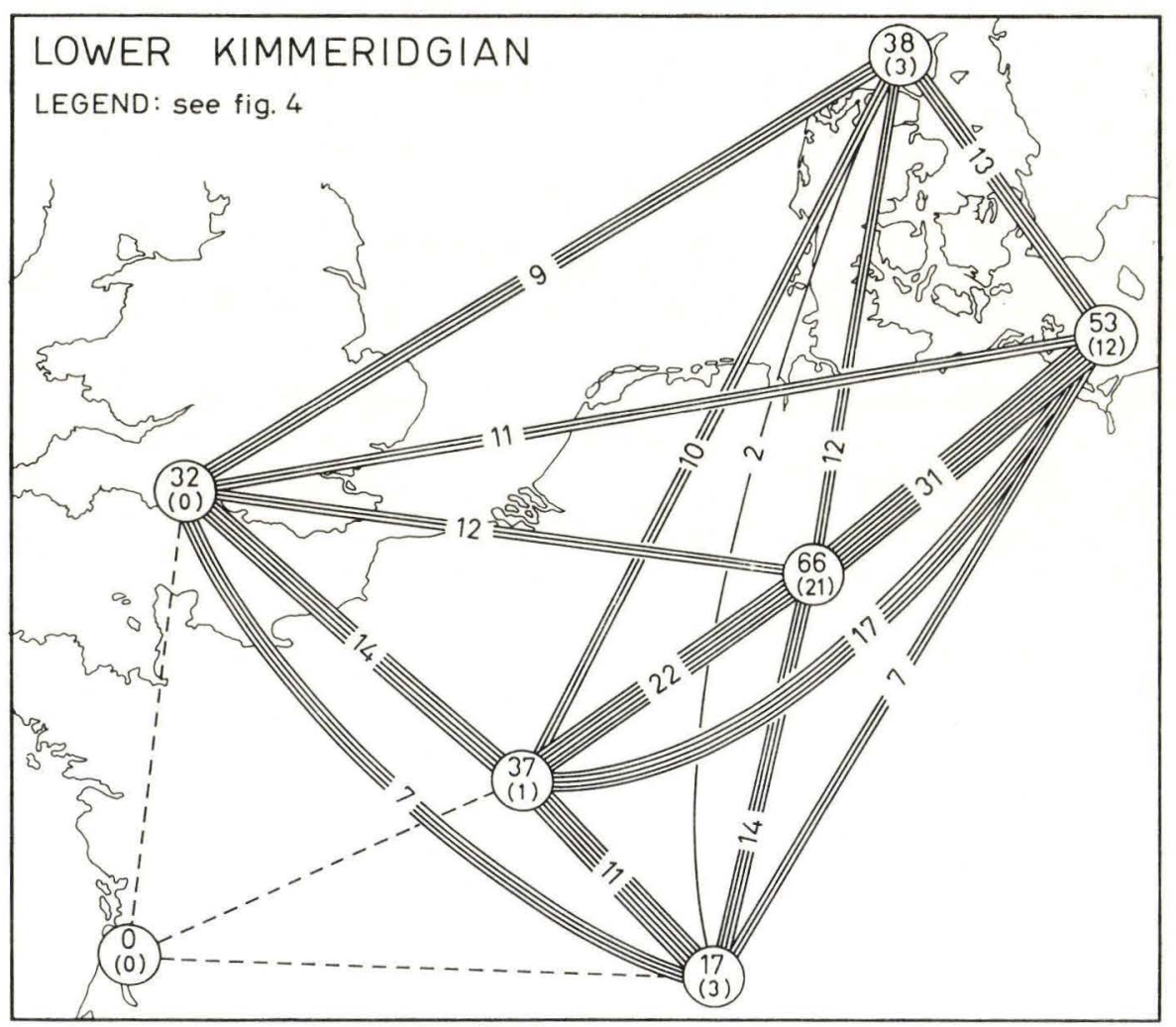

Fig. 5. 


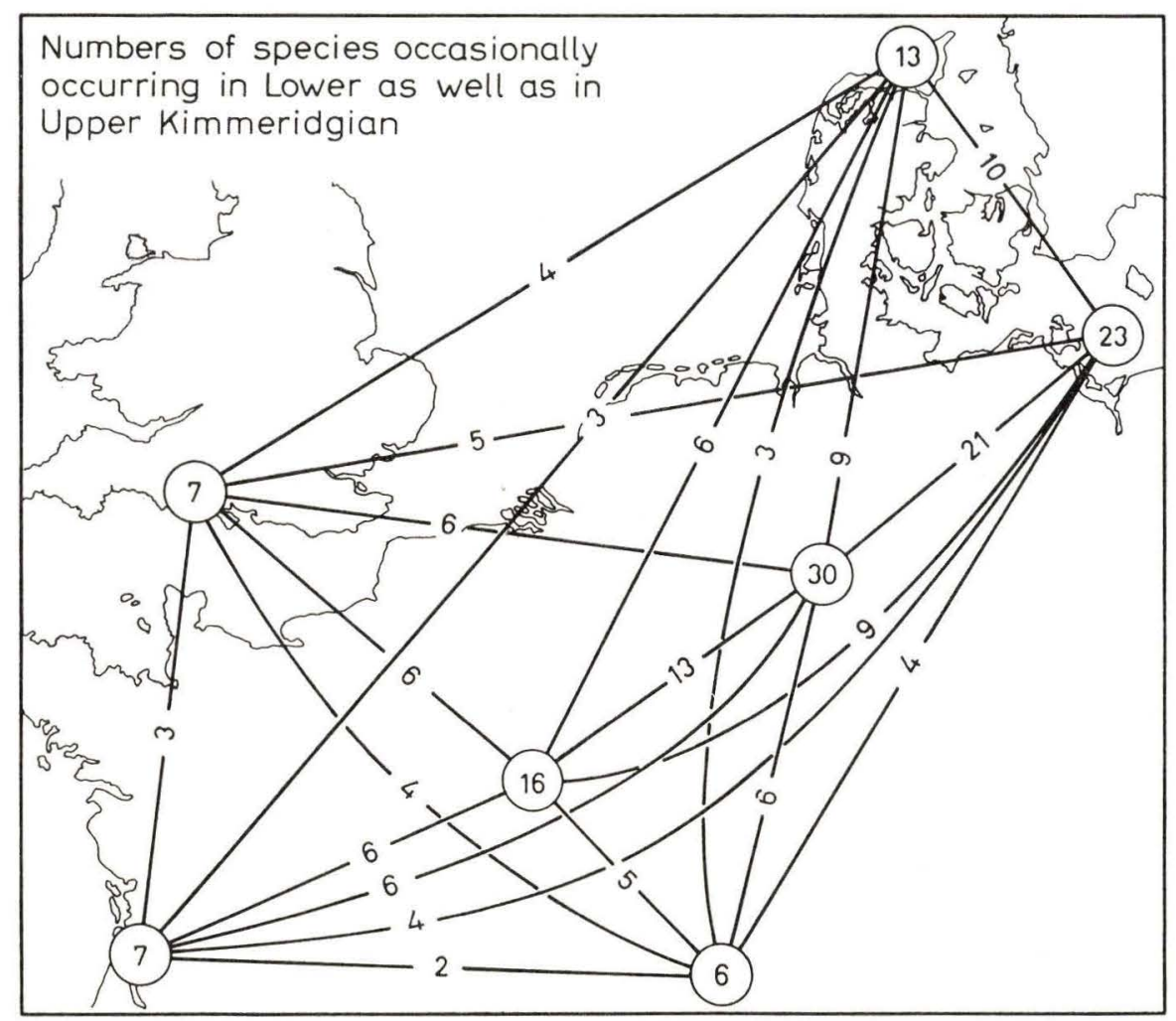

Fig. 6.

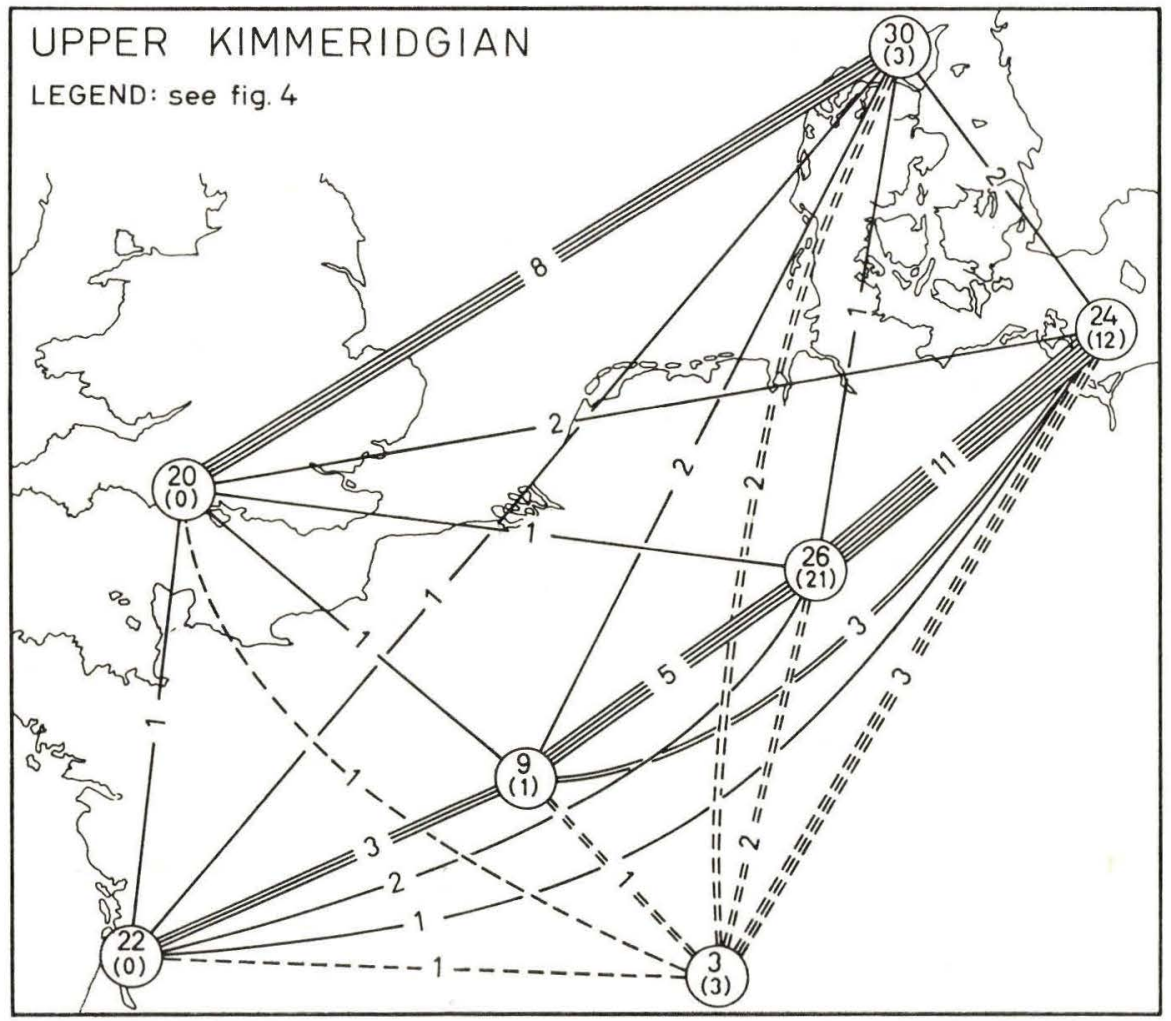

Fig. 7 . 
when, comparing the number of species of the different areas of deposition, those species which at one or more places have been found both in the Upper and the Lower Kimmeridgian are excluded (fig. 6, p. 37). This may perhaps be due to the uncertainty in the exact placing of the boundary between the Upper and the Lower Kimmeridgian in these areas, but is probably more likely to be due to the fact that in the areas of the North Sea Basin there are no sequences of ostracod faunas showing continuity of development between Lower and Upper Kimmeridgian.

Thus, for instance in the Dorset area there are no species in the ostracod faunas in common to both Lower and Upper Kimmeridgian.

Several of those species which in the deposits of the North Sea Basin are found in the Upper Kimmeridgian seem to be closely related to or identical with species which in the Mid-European Region of deposition are found mainly in the Lower Kimmeridgian. For example species of Macrodentina, which occur throughout the whole of the Kimmeridgian in the Mid-European Region, a few species of this genus found in the entire Kimmeridgian of the North Sea Basin show close affinity to species from the Lower Kimmeridgian of Northwest Germany. The genus Mandelstamia is - with one exception - found only in the North Sea Basin. This exception is the species Mandelstamia inflata, which is found only in the Mid-European Region. The genus Galliaecytheridea is widely distributed in the Lower Kimmeridgian over the entire area of Northern and Western Europe. It is also common in the North Sea Basin throughout the Upper Kimmeridgian and the Portlandian. But in the Mid-European Region, species of this genus have been found only early in the Upper Kimmeridgian. Species of the genera Cytherella and Cytherelloidea are only scantily represented in the Kimmeridgian of the North Sea Basin, and in the North West Danish Embayment none have been found. This effective faunal barrier between the North Sea Basin and the Mid-European Region originating at the time of transition from Lower to Upper Kimmeridgian is probably due to several factors.

There are facies differences between the two regions. The deposits of the Mid-European Region consist to a large extent of limestones, while the deposits in the North Sea Basin are developed mainly as clayey sediments, usually non-calcareous. Furthermore, many of the deposits in the MidEuropean Region are more or less brackish or limnic. These differences in the conditions of deposition are, however, not sufficient to explain the mutually exclusive nature of the Upper Kimmeridgian ostracod faunas of the two regions. A physical barrier in the form of land between the two areas of deposition might explain the great differences in the faunas as well as in the lithological development. Such an area of land (Kimbria) could, during some short intervals in the Kimmeridgian and Portlandian have been overcome by the sea. Such a small marine ingression took place once 
in the Danish Embayment in the Upper Portlandian (Lower Purbeckian), forming the only direct marine connection between the North West Danish Embayment and the North West Polish Basin during the time interval from Lower Kimmeridgian to Hauterivian (BRUUn Christensen 1970).

The ostracod faunas of the Upper Kimmeridgian of the North Sea Basin show some affinities to faunas described from the Volga-Ural region of the U.S.S.R. (Lubimova, 1955) including the type section of the Volgian Stage (Boreal Realm). Chronostratigraphical correlation of the Volgian with the Upper Kimmeridgian of the North Sea area is, however, impossible at. present as biostratigraphical evidence is largely lacking and chronostratigraphical correlations over such distances are not possible without convincing evidence. Possible future discoveries of genuine chronostratigraphical evidence would solve this difficulty. The uncertainty in the correlation of biostratigraphical units of the areas considered with the units of the Kimmeridgian Stage in Dorset, and the use of this as a standard for a geological time is, however, considerably smaller than the uncertainty would be by the similar use of the Volgian Stage in a completely different basin at a much greater distance.

The possibilities of chronostratigraphical correlation are in this case presumably more to be sought among physical rather than among biological factors. It is possible that an extensive boreal inland sea was in existence in the Upper Jurassic (Hallam 1969). A study of the conditions connected with this hypothesis may open the possibility of using as chronostratigraphical evidence certain regionally widespread physical changes in the sea which left their marks directly on these deposits (such as eustatic movements, sudden temperature changes or changes in salt content etc.). 


\title{
DESCRIPTION OF SPECIES
}

\author{
SUBCLASSIS OSTRACODA LATREILLE 1806
}

Ordo Podocopina Müller 1894

Familia Cytherellidae SARS 1866

Genus Cytherelloidea AleXANDER 1929

Cytherelloidea paraweberi OERTLI 1957

partim 1951 Cytherelloidea weberi Steghaus, p. 207, pl. 14 figs. 3, 5 (non figs. 4-6).

?partim 1955 Cytherelloidea weberi Steghaus - Schmidt, p. 51.

1957 Cytherelloidea paraweberi OERTLI, p. 65, pl. 1 figs. 12-15.

1959 Cytherelloidea paraweberi OenTLI-OentLI, p. 18, pl. 2 figs. 26-27.

1964 Cytherelloidea paraweberi OertLI-GLashoff, p. 52.

non 1966 Cytherelloidea paraweberi OenTLI-BARKer, p. 457, pl. 3, figs. 7-9, p. 485 , pl. 9, figs. $1-2$.

1968 Cytherelloidea paraweberi OeRTLI-Bielecka \& StyK, fig. 2.

1969 Cytherelloidea paraweberi OertLi-Kilenyi, p. 116, pl. 23, figs. 8-9, textfig. 5a.

Diagnosis

Carapace quadrangular. Each valve with a peripheral continous ridge parallel and equidistant from the margin. A curved median rib branches out from the upper one, descends to the middle of the valve, where it turns to run parallel with dorsal margin.

Remarks

The specimen illustrated by BAKER (1966 figs. 7-9) differs too much from C. paraweberi OeRtLI to be included in this species. It is much thicker in dorsal aspect, especially in the posterior part of the carapace.

\section{Distribution}

DoRset: In Galliaecytheridea elongata Zone, Kimmeridge Clay.

North Germany: Unteres Kimmeridge.

NW. Poland: Astart and dolny kimeryd.

PARIS BASIN: Kimméridgien inférieur (moyen).

Swiss Jura: Mittleres-Oberes Kimmeridge. 
Familia Schulerideidae MANDELSTAM 1959

Subfamilia Schulerideinae MANDELSTAM 1959

Genus Schuleridea Swartz \& SwaIn 1946

Schuleridea (Schuleridea) moderata nov. sp.

Plate I figs. 1a-i

1969 Schuleridea sp. 1 KILENYI, p. 119, pl. 23, figs. 34-39.

1969 ?Schuleridea sp. 2 KILENYI, p. 119, pl. 24 fig. 1.

(MS) Schuleridea (Schuleridea) moderata BRUUN CHRISTENSEN \& KILENYI - BRUUN Christensen (MS)

Derivation of Name. From Latin moderatus, moderate.

Holotype. A carapace; Plate I, figs. 1a-b. D.G.U. Catalogue No. 1970 - OBC - 1. Type Locality. Borehole Fjerritslev No. 1, D.G.U. File No. 24.216.

Type Stratum. Dark claystone; 474-480 m, $147 \mathrm{~cm}$ below the top of the core. Børglum Formation. Upper Kimmeridgian.

Material in the Sample. Nine carapaces, three valves and fragments.

\section{Diagnosis}

A weakly but densely punctate species of Schuleridea (Schuleridea) with rounded periphery and a weak overlap by the left valve. The posterodorsal margin is rounded, long, and relatively steep with the rounded posterior end near the ventral margin and a weakly developed posterodorsal angle less than fourth of the valve's length from the posterior end.

\section{Affinities}

The species is closely related to Schuleridea n.sp. aff. triebeli (STEGHAus) in MALz (1958a, pl. 2 fig. 14-16), but the dorsal margin on the left valve is more flat and there are fewer porecanals in the marginal zone.

\section{Description}

The carapace is oviform. The anterior margin is circular, rounded. The ventral margin is straight to weakly convex. In the posterior part, the ventral margin curves upwards towards the rounded posterior end, situated in the lowermost fourth of the valves in females. In male valves the posterior end is nearly circularly rounded into the posterodorsal margin, with continued smoothness into the dorsal margin. Above the posterior end the posterodorsal margin on females has a small convexity to the posterior cardinal angle about one fourth of the valve's length from the posterior end, and one fourth of the valve's height from the greatest height of the carapace. The dorsal margin on the females is nearly semicircular. The greatest height is situated just anterior of the middle of the carapace. The overlap of the left valve is nearly the same along the whole periphery. In dorsal aspect the lateral surfaces are smoothly arched, and the carapace is pointed terminally. 
The lateral surfaces are finely and densely punctate. The eye spot is relatively weakly developed. The anterior marginal zone contains 20-30 radial pore canals, mostly straight, only in the uppermost part a few are bent upwards. In the posterior marginal zone 12-15 radial porecanals have been found. The hinge and the muscle scars as in other species of the genus, but the accomodation groove is very weakly developed.

$\begin{array}{lcc}\text { Measurements: } & \text { Length } & \text { Height } \\ \text { Females } \ldots \ldots \ldots \ldots \ldots \ldots \ldots \ldots \ldots & 0.65-0.67 \mathrm{~mm} & 0.43-0.44 \mathrm{~mm} \\ \text { Males } \ldots \ldots \ldots \ldots \ldots \ldots \ldots & 0.78 \mathrm{~mm} & ? 48 \mathrm{~mm}\end{array}$

\section{Distribution}

North West Danish Embayment: Børglum Formation, Upper Kimmeridgian.

South England: Kimmeridge Clay, Mandelstamia (X.) maculata Zone and the lowermost part of Galliaecytheridea spinosa Zone, Upper Kimmeridgian in Dorset. Smaller specimens of the species have been found in a sample from the Kimmeridge Clay, Cumnor Hills near Oxford.

\section{Genus Galliaecytheridea OERTLI 1957}

\section{Galliaecytheridea dissimilis OeRTLI 1957 s. 1.}

1957 Galliaecytheridea dissimilis OeRTLI, p. 655, pl. 1 figs. 32-39, pl. 2 figs. 40-44.

1964 Galliaecytheridea dissimilis OertLI - GlashofF, p. 38, pl. 4, figs. 8-11.

1968 Galliaecytheridea dissimilis OeRTLI - BIELECKA \& STYK, fig. 2.

1969 Galliaecytheridea dissimilis Oertli - KILENYI, p. 120, pl. 24, figs. 8-20, textfigs. $3 \mathrm{a}, 5 \mathrm{~d}$.

(MS) Galliaecytheridea dissimilis OeRTLI - BRUUn CHRISTENSEN (MS).

\section{Original Diagnosis}

"Espece du genre Galliaecytheridea avec les caractéristiques suivantes: inégalité frappante des valves: valve gauche beaucoup plus haute sur les parties dorsale et ventrale. Arriere triangulaire assez pointu, surtout pour la valve droite. Surface finement ponctuée". (OERTLi 1957, p. 655).

\section{Remarks}

This species occurs in the Dorset profile as two morphological subspecies. The stratigraphically higher specimens of $G$. dissimilis (large var.) are characterized by their size, and by rhomboidal outline caused by the development of a postero-ventral bulge. Some specimens from the Danish Embayment have this posteroventral bulge in a very strongly developed form. In the lowermost part of the Kimmeridge Clay G. dissimilis s. str. has been found. 


\section{Distribution}

PARIS BASIN: Oxfordien supérieur ("Séquanien") to partie inférieure du Kimméridgien inférieur.

DORSET: Upper Oxfordian to Lower Kimmeridgian (Galliaecytheridea dissimilis s. str. Zone and G. elongata Zone in Kimmeridge Clay).

N. German Basin: Mittlerer Korallenoolith to unterer Kimmeridge.

NW. Polish Basin: Orgow \& Raurak \& Astart (Oksford) and dolny kimeryd.

NW. Danish Embayment: Lowermost Børglum Formation (Uppermost Oxfordian and Lowermost Kimmeridgian).

\section{Galliaecytheridea compressa nov. sp.}

Plate I figs. 2a-f, plate II figs. 1a-d

1966 Galliaecytheridea wolburgi (STEGHAUs) - BARKER, p. 450, pl. 2 figs. 1-8.

(MS) Galliaecytheridea compressa BRUUN CHRISTENSEN \& KilenYI - BRUUn ChriSTENSEN (MS).

Derivation of Name. From Latin compressus, compressed.

Holotype. A female carapace, plate I figs. 2a-b. No IO 2763 deposited in the British Museum (Natural History) (BARKER's collection).

Type Locality. Hounstout Cliff, Dorset.

Type Stratum. Bed 7, Portland Sand.

Diagnosis

A punctate species of Galliaecytheridea with the central valve surfaces more or less concave seen in dorsal view. The central part of the posterodorsal margin has a slight but characteristic angle with an occas:onally developed posteroventral spine in the otherwise evenly rounded curve.

\section{Affinities}

The species has to be separated from $G$. wolburgi (STEGHaus) by the concavity of the central part of the valves, and from $G$. crendonensis BARKER and $G$. spinosa KILENYI by having an angle near the middle of the nearly circularly curved posteroventral margin, and considerably less pointed posterior ends. On G. wolburgi (Steghaus) there is a distinct angle between the rather straight posteroventral margin and the straight ventral margin. G. polita KILENYI lacks the central compression of the valves and is not punctate.

\section{Distribution}

DorseT: The species has been found in samples from the entire lower part of the type section of the Portlandian (Portland Sand) and perhaps 
from the uppermost part of the Kimmeridge Clay just below the Massive Bed and the Black Nore Sandstone:

"Samples from beds $10 \mathrm{ft}$. below the Massive Bed to the Lower Parallel Band at Hounstout Cliff have yielded a characteristic ostracod fauna, as also have beds at West Weare Cliff from $10 \mathrm{ft}$. below the Black Nore Sandstone to the Basal Shell Bed". (BARKer 1966, pp. 447448).

NW. Danish Embayment: Uppermost Børglum Formation (Portlandian).

Galliaecytheridea dorsetensis nov. sp.

Plate II figs. 3a-d, ? 4a-d

part. 1957 Cyprideis wolburgi (Steghaus) - Oertli, p. 657, pl. 2 figs. 56-58 (non fig. 59-60), (non pl. 3 fig. 61-68).

part. 1968 Galliaecytheridea wolburgi (STEGHAUS) - BRUUn CHRISTENSEN.

1969 Galliaecytheridea wolburgi (STEghaus) - KILENYI, p. 121, pl. 24 figs. 21-27.

(MS) Galliaecytheridea dorsetensis BRUUn CHRISTENSEN \& KILENYI - BRUUn CHRISTENSEN (MS)

Derivation of Name. From Dorset, South England.

Holotype. A left valve. No HU 2.J.13.1 (KILENYI 1969, pl. 24 fig. 21) Geology Department- University of Hull.

Type Locality. Black Head, Dorset.

Type Stratum. Galliaecytheridea dissimilis Zone (Zone of Rasenia cymodoce) about ten feet above Ringstead Coral Bed, Kimmeridge Clay (Lowermost Kimmeridgian) .

Material. 347 valves and carapaces from Kimmeridge Clay, Dorset.

Diagnosis

A punctate species of the genus Galliaecytheridea with convex ventral margin, which curves smoothly into the posteroventral margin. The dorsal margin is straight with a slight concavity in the posterior half and a gently convex anterior part especially in the left valve. Greatest height is situated near the middle of the carapace.

\section{Affinities}

The species has to be separated from $G$. wolburgi s. str. by the convex ventral margin. In $G$. wolburgi s. str. the ventral and dorsal margins are nearly straight and parallel. The juvenile carapaces are difficult to distinguish from specimens of G. minuta, G. elongata or G. postrotunda.

\section{Distribution}

Dorset: Galliaecytheridea dissimilis Zone and G. elongata Zone, Lower Kimmeridge Clay.

PARIS BASIN: Kimméridgien inferieur. 
NW. Polish Basin: Amphicythere (A.) semisulcata - Schuleridea (S.) triebeli Fauna, Fyledal Clay, Lower Kimmeridgian.

\section{Galliaecytheridea elongata KILENYI 1969}

1969 Galliaecytheridea elongata KILENYI, p. 123, pl. 25 figs. 6-12.

1969 Galliaecytheridea trapezoidalis KILENYI, p. 123, p1. 25, figs. 13-19, textfigs. 3f, $5 \mathrm{~g}$. (MS) Galliaecytheridea elongata KILENYI - BRUUN CHRISTENSEN (MS).

Diagnosis

A relatively small punctate species of Galliaecytheridea with slightly concave posterodorsal margin and slightly pointed posterior end in the horizontal median of the carapace. Left valve overlaps clearly the right one dorsally and ventrally. The anterior margin is evenly curved and more flattened in the dorsal part, especially on the female carapace. The dorsal and ventral margins on left valves of the latter are curved slightly, tapering from the mid-line of the carapace, where the largest height is situated. Carapace of males is long with nearly straight and parallel ventral and dorsal margins.

\section{Remarks}

The females of this species were described by Kilenyi (1969) as G. trapezoidalis.

\section{Distribution}

Dorset: G. elongata Zone, Lower Kimmeridge Clay.

NW. Danish Embayment: Børglum Formation, Lower Kimmeridgian.

\section{Galliaecytheridea gracilis GLASHOFF 1964}

Plate II figs. $2 \mathrm{a}-\mathrm{b}$

1964 Galliaecytheridea gracilis GLASHOFF - p. 39, pl. 4 figs. 12-18.

1969 Galliaecytheridea sp. 2 KILENYI, p. 131, pl. 27 figs. 1-4.

(MS) Galliaecytheridea gracilis GLASHOFF - BRUUN CHRISTENSEN (MS)

Diagnosis

A small species of the genus Galliaecytheridea with nearly rounded posterior end and convex ventral margin. The anterior margin is evenly curved ending at the largest height of the carapace, a third $(q)$ or a fourth $\left(O^{7}\right)$ of the length of the carapace from the anterior end. Posterior part of dorsal margin is straight.

\section{Distribution}

DORSET: Upper Oxfordian and Lowermost Kimmeridgian (G. dissimilis Zone). 
N. German Basin: Oberer Korallenoolith to Basis Kimmeridge.

NW. Danish Embayment: Frederikshavn and Børglum Formations, Lower

Kimmeridgian.

\section{Galliaecytheridea oertlii nov. sp.}

Plate III figs. 1a-i

part. 1957 Galliaecytheridea wolburgi Steghaus-Oertli, p. 657 pl. 3 figs. $63-68$ (non pl. 2 figs. 56-60 or pl. 3 figs. 61-62, 66).

part. 1968 Galliaecytheridea wolburgi STEGHAUs-BRUUn CHRISTENSEN.

(MS) Galliaecytheridea oertlii BrUUn Christensen \& KILENYI - BRUUn ChriSTENSEN (MS).

Derivation of Name. In honour of Dr. H. J. Oertli, Pau, France.

Holotype. A female carapace. Plate III figs. 1b-c. D.G.U. Catalogue No. $1970-$ $\mathrm{OBC}-18$.

Type Locality. Borehole Øresund (The Sound) No. 1A near Elsinore, D.G.U. File No. 188.345 .

Type Stratum. Sample No. 23, core $51.70-53.15 \mathrm{~m}, 73 \mathrm{~cm}$ below top of the core. Fyledal Clay (Beds with clay and limestone, dark grey, greenish and bluish, hard, shelly Lower part of Mandelstamia inflata Zone (BRUUN CHRISTENSEN MS) of Lower Kimmeridgian age.

\section{Material}

Several hundred valves from the type stratum.

\section{Diagnosis}

A smooth or very weakly punctate species of Galliaecytheridea with pointed posterior end and very slightly convex ventral margin. Anterior margin is almost semicircular. The greatest height is about one-fifth of the valve's length from the anterior, near the anterior cardinal angle. The dorsal margin is straight. The posterior cardinal angle is well marked and is situated near the posterior end. The posterodorsal margin is long and straight in females and is short and rounded in males. In dorsal view the carapace is almost elliptical in shape, lacking any central concavity. The larval stages have commonly a posteroventral spine which is rarely present in adults.

\section{Remarks}

This species is close to Paleocytheridea grigorievi Ljubimova (1958, p. 561, pl. III fig. 4) from the Lower Kimmeridgian of the U.S.S.R.

\section{Distribution}

NW. Polish Basin: Zone of Mandelstamia inflata (Bruun Christensen MS), Fyledal Clay, Lower Kimmeridgian. Scania.

Paris Basin: Kimméridgien inferieur. 


\section{Galliaecytheridea polita KILENYI 1969}

1969 ?Galliaecytheridea polita KILENYI, p. 128, pl. 26, figs. 21-26, textfig. 36.

(MS) Galliaecytheridea polita KILENYI - BRUUN CHRISTENSEN (MS).

\section{Diagnosis}

A smooth species of Galliaecytheridea with the left valve only slightly larger than the right one. Dorsal and ventral margins nearly straight and parallel. The curved posteroventral margin has a slight angle at the central part at least on female left valves. In dorsal view the lateral surfaces are smooth and convex.

\section{Affinities}

The species is very close in outline to $G$. compressa nov. sp., which is characterised by punctate valve surfaces and lateral compression in the central part of the valve.

\section{Remarks}

Valves from the Danish material show that the species has to be classified as a true Galliaecytheridea.

\section{Distribution}

Dorset: Galliacytheridea polita Zone, Uppermost Kimmeridge Clay.

NW. Danish Embayment: Uppermost Børglum Formation or lowermost Vedsted Formation, Uppermost Kimmeridgian and Portlandian.

\section{Galliaecytheridea postrotunda OERTLI 1957}

1957 Galliaecytheridea postrotunda OERTLI, p. 656, pl. 2 figs. 45-55.

1959 Galliaecytheridea postrotunda OERTLI - OERTLI, p. 25, pl. 3, fig. 89.

1964 Galliaecytheridea postrotunda OERTLI - GLASHOFF, p. 38, pl. 4, figs. 4-7.

non 1966a Galliaecytheridea postrotunda OERTLI - BARKER, p. 450, pl. 3, figs. 1-6.

1969 Galliaecytheridea postrotunda OeRTLI - KILENYI, p. 129, pl. 27, figs. 5-14, textfigs $3 ; 5 \mathrm{k}$.

1969 Galliaecytheridea malzi KILENYI, p. 123, pl. 25 figs. 20-22, textfigs. 3 k, 5 f. (MS) Galliaecytheridea postrotunda OERTLI - BRUUn CHRISTENSEN (MS).

\section{Original diagnosis}

"Espéce du genre Galliaecytheridea avec les caractèes suivants: contour latéral en forme d'ovale allongé. Arrière bien arrondi; point extrême à mi-hauteur ou un peu au-dessous. Angle cardinal postérieur bien marqué. Bord antérieur souvent avec plusieurs dents fines. Épine sur la valve droite près du bord postéroventral. Surface ponctuée, peu dans le milieu, avec une grande densité dans la partie ventrale et les extrémités." (OerTLi 1957, p. 656). 


\section{Distribution}

PARIS BASIN: Oxfordien supérieur to Kimméridgien moyen.

DoRseT: Corallian to Lower Kimmeridge Clay (Lower part of the Zone of $G$. dissimilis s. str.)

NW. Polish Basin: Upper Oxfordian to Lowermost Kimmeridgian (Argow to dolny kimeryd).

N. German Basin: Unterer Kimmeridge.

NW. Danish Embayment: Haldager Formation and Børglum Formation (Upper Oxfordian and Lower Kimmeridgian).

\section{Galliaecytheridea spinosa KILENYI 1969 \\ Plate III figs. $2 \mathrm{a}-\mathrm{c}$; textfig. 8}

1969 Galliaecytheridea spinosa KILENYI, p. 128, pl. 26, figs. 10-20.

1969 Galliaecytheridea sp. 3 KILENYI, p. 132, pl. 27 figs. 15-16.

(MS) Galliaecytheridea spinosa KILENYI - BRuUn Christensen (MS).

\section{Diagnosis}

Species of Galliaecytheridea with extremely pointed posterior end. Two or three little spines usually on posterior extremity. Sexual dimorphism very strong, males much longer than females. Surface of valve pitted.

\section{Remarks}

This species is close to Galliaecytheridea crendonensis BARKER 1966 but in $G$. spinosa the dorsal and ventral margins are nearly parallel, whereas in $G$. crendonensis these margins taper strongly towards the posterior. $G$.

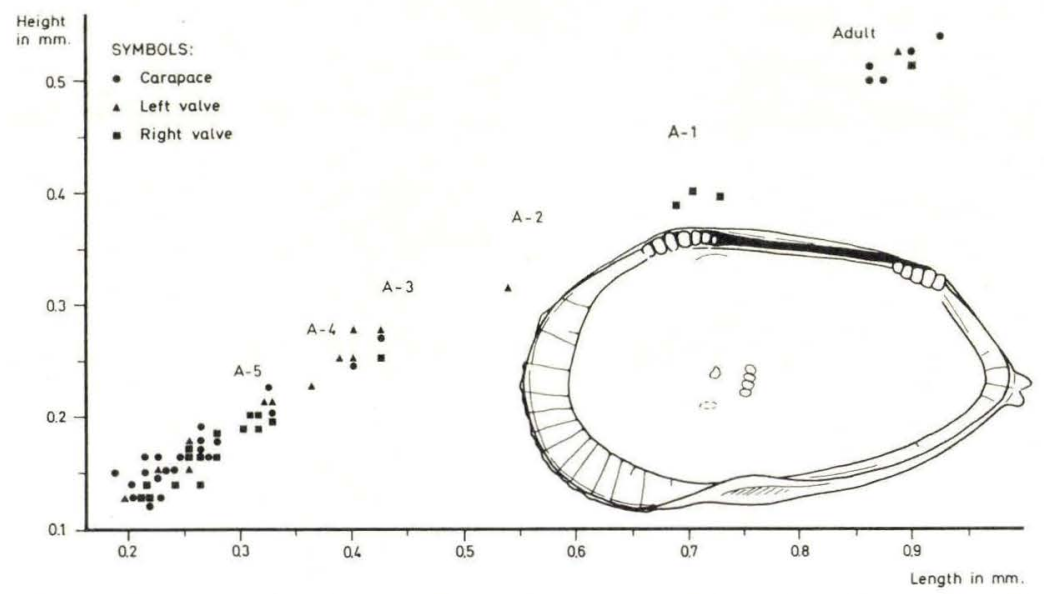

Fig. 8. Scattergram showing relations between numbers, lengths, and heights of Galliaecytheridea spinosa KILENYI $s$. $l$. from boring Fjerritslev No. 1, core 474-480 m, $147 \mathrm{~cm}$ below the top of the core. Outline of the adult right valve from sample shown. 
crendonensis has also a more strongly developed caudal process, which is in a posteroventral position and not at mid-height as in G. spinosa.

\section{Distribution}

Dorset: G. spinosa Zone, Upper Kimmeridge Clay.

NW. Danish Embayment: Børglum and Frederikshavn Formations, Upper Kimmeridgian.

\section{Galliaecytheridea wolburgi (STEGHAUS 1951)}

1951 Cyprideis wolburgi Steghaus, p. 213, pl. 14 figs. 24-25, pl. 15 fig. 16.

1955 Cyprideis wolburgi wolburgi (Steghaus) - Schмidt, p. 58, pl. 2 figs. 25-26.

?part.1957 Galliaecytheridea wolburgi (Steghaus) - Oertli, p. 657, pl. 2 figs. 61-62, 66 (non pl. 2 figs. 56-60, pl. 3 figs. 63-65).

1962 Galliaecytheridea wolburgi (Steghaus) - Klingler, Malz \& Martin, p. 176, pl. 26 fig. 15, pl. 27 fig. 19.

1964 Galliaecytheridea wolburgi (Steghaus) - Glashoff, p. 39, pl. 4 figs. 1-3.

non 1966 Galliaecytheridea wolburgi (STEGHAUS) - BARKER, p. 450, pl. 2 figs. 1-8.

non 1968 Galliaecytheridea wolburgi (Steghaus) - BruUn Christensen, pl. 23 fig. 76.

non 1969 Galliaecytheridea wolburgi (Steghaus) - KILENYI, p. 121, pl. 24 figs. 21-27, text figs. $3 \mathrm{c}, 5 \mathrm{c}$.

? (MS) Galliaecytheridea cf. wolburgi (Steghaus) - BruUn Christensen (MS).

\section{Diagnosis}

A large species of Galliaecytheridea with long and parallel dorsal and ventral margins. The posterior end lies well above the mid-height of the valve. The posteroventral margin is straight, steep and long with well marked angle to the ventral margin.

\section{Remarks}

A number of species have been described under the name of G. wolburgi and it is very difficult to clear up the present confusion without examining the material in question. G. wolburgi minuta (ScHMIDT 1955) may well be a separate species and has been considered as such in the present paper (see plate II figs. 5a-b).

\section{Subfamilia Cuneocytherinae Mandelstam 1960}

Genus Dicrorygma POAG 1962

Subgenus Dicrorygma (Orthorygma) BRUUn Christensen 1965

Dicrorygma (Orthorygma) reticulata BRUUN CHRISTENSEN 1965

1957 Cytherideinarum gen.? sp. 1 OerTLI, p. 661, pl. 3 figs. 86-91.

1965 Dicrorygma (Orthorygma) reticulata BRUUn CHRISTENSEN, p. 17, pl. 1 figs. 6a-f, pl. 2 figs. $5 \mathrm{a}-\mathrm{f}$.

1965 Oertliana kimmeridgensis KILENYI, p. 573, pl. 79 figs. 1-12, textfig. 1. 
1969 Dicrorygma (Orthorygma) kimmeridgensis (KILENYI) - KILENYI, p. 137, pl. 31 figs. $22-31$.

(MS) Dicrorygma (Orthorygma) reticulata BRUUn CHRISTENSEN - BRUUN CHRISTENSEN (MS),

\section{Original Diagnosis}

"A reticulate species of Dicrorygma (Orthorygma) with almost straight posterodorsal margin. The posterior angle is rounded and situated in one fourth of the length of the valve from the posterior end." (BRUUN CHRISTENSEN 1965, p. 17).

\section{Distribution}

NW. Danish Embayment: Zone of Galliaecytheridea spinosa, Børglum

Formation. Upper Kimmeridgian (considered to be Lower Kimmeridgian in BruUn Christensen 1965), and in samples from lower levels.

Dorset: Zone of Galliaecytheridea elongata, Lower Kimmeridge Clay.

PARIS BASIN: Lower Kimmeridgian (Kimméridgien infèrieur (inf.-moy.))

\section{Dicrorygma (Orthorygma) brotzeni BRUUN CHRISTENSEN 1965}

1955 Ostracod 102 Schмid, p. 61, pl. 3A figs. 39-40.

1961 Cytherideinarum gen. sp. 2 Oertli, Brotzen and Bartenstein, p. 13, pl. 1 figs. $3 \mathrm{a}-\mathrm{b}$.

1965 Dicrorygma (Orthorygma) brotzeni BruUn Christensen, p. 19, pl. 1 figs. 8a-d, pl. 2 figs. $4 a-d$.

1965 Oertliana sp. 1 KilenYi p. 574, pl. 79 figs. 13-16.

1968 Dicrorygma (Orthorygma) brotzeni BRUUn CHRISTENSEN pl. 23 fig. 88.

1968 Dicrorygma (Orthorygma) sp. WienHolz, pl. 4 fig. 42.

1969 Dicrorygma (Orthorygma) sp. 1 (KilenYI) - KilenYI, p. 137, pl. 31 figs. 32-33.

(MS) Dicrorygma (Orthorygma) brotzeni BRUUn Christensen (MS).

Original diagnosis

"A dense punctate species of Dicrorygma (Orthorygma) with slightly convex dorsal margin, slightly developed cardinal angle and approximately rounded posterior termination". (BRUUn Christensen 1965, p. 19).

\section{Remarks}

The species has an exterior outline very reminiscent of Mandelstamia ignobilis LuUbimova 1955 as this has been described and figured by LJUBIMOVA (1955, p. 66, pl. 7 figs. 3a-b) from the Lower Volgian in the Buzuluk Region of the U.S.S.R. The description includes no morphological feature diverging from that of $D$. (O.) brotzeni. 
Distribution

NW. Polish Basin: Common in deposits of Upper Kimmeridgian Age. In Scania the species occurs in the Zone of Mandelstamia inflata from uppermost Lower Kimmeridgian and from Upper Kimmeridgian deposits (Bruun Christensen MS). In northeastern Germany the species seems to occur in deposits of Portlandian Age (Wienholz 1968).

Dorset: Zone of Galliaecytheridea spinosa Kilenyi, Upper Kimmeridge Clay.

North German Basin: Kimmeridge.

Familia Loxoconchidae SARS 1925

Genus Mandelstamia LJubimova 1955

\section{Mandelstamia (Mandelstamia) rectilinea MALZ 1958}

1958 Mandelstamia rectilinea MaLz, p. 38, p1. 11 figs. 58-63.

1961 Mandelstamia rectilinea Malz - Neale and Kilenyi, p. 440, pl. 71 figs. 1-4, 6.

1961 Mandelstamia triebeli KILENYI - NeAle and KILENYI, p. 442, pl. 71 figs. 5, 9-10, 14-15.

1964 Mandelstamia rectilinea MALZ - GLASHoff, p. 48.

1969 Mandelstamia (Mandelstamia) rectilinea MALZ - KILENYI, p. 133, pl. 29 figs. 1-6, textfigs. $4 \mathrm{a}, 6$.

1969 Mandelstamia (Mandelstamia) triebeli KILENYI - KilenYI, p. 133, pl. 29 figs. 9-10, textfig. $4 \mathrm{c}-\mathrm{d}, 5 \mathrm{k}$.

(MS) Mandelstamia (Mandelstamia) rectilinea MALZ - BRUUn CHRISTENSEN (MS).

Original diagnosis

"Eine Art der Gattung Mandelstamia mit folgenden Besonderheiten: Seitenflächen mit grossen, rundlichen Grübchen besetzt. In Dorsalansicht ist die Wölbung der Seitenflächen durch einen flachen Randstreifen vom Vorderrand abgesetzt, die grösste Breite liegt hinter der Mitte, am Hinterende stossen die Seitenflächen in einem spitzen Winkel zusammen. Sexual-Dimorphismus ausgeprägt.” (MALz 1958 p. 38).

\section{Remarks}

The strongly developed sexual dimorphism of the species, with large males, having inflated carapace, with often complicated reticulation on the posterior part can be observed on all the specimens in the Danish Embayment.

\section{Distribution}

Dorset: Zones of Galliaecytheridea dissimilis and G. elongata, Lower Kimmeridge Clay, and probably in Oxfordian.

NW. Danish Embayment: Børglum Formation. Lower Kimmeridgian (Zones of $G$. dissimilis and G. elongata). 


\section{Subgenus Mandelstamia (Xeromandelstamia) \\ BEUTLER and GRÜNDEL 1963}

Mandelstamia (Xeromandelstamia) tumida nov. sp.

Plate IV figs. 1a-d; textfig. 9.

1961 Mandelstamia sp. 1 Kilenyi - NeAle and Kilenyi, p. 446, pl. 71 figs. 7-8, 13.

1969 Mandelstamia (Xeromandelstamia) sp. 1 KILENYI - KILENYI, p. 136, pl. 29 figs. 7-8.

(MS) Mandelstamia (Xeromandelstamia) tumida BRUUn Christensen and KILENyI BRUUN CHRISTENSEN (MS).

Derivation of Name. Of Latin tumidus, swollen.

Holotype. A carapace. Plate III figs. 3e-f. D.G.U. Catalogue No. 1970 - OBC - 31. Type Locality. Borehole Fjerritslev No. 1, D.G.U. File No. 24.216.

Type Stratum. Dark claystone; $474-480 \mathrm{~m}, 147 \mathrm{~cm}$ below the top of the core. Børglum Formation. Upper Kimmeridgian.

Material in the Sample. Seven carapaces and 21 valves.

\section{Diagnosis}

A reticulate species of Mandelstamia (Xeromandelstamia) with nearly parallel dorsal and ventral margins and with a posterodorsal tubercle, situated one-third of the valve's length from the posterior cardinal angle. A second, less strongly developed but elongated posterovental tubercle may also be present. These tubercles are less conspicuous in the adult stage than in instars. In the males the individual pits of the reticulate ornamentation are subdivided. In females the ornamentation is uniform.

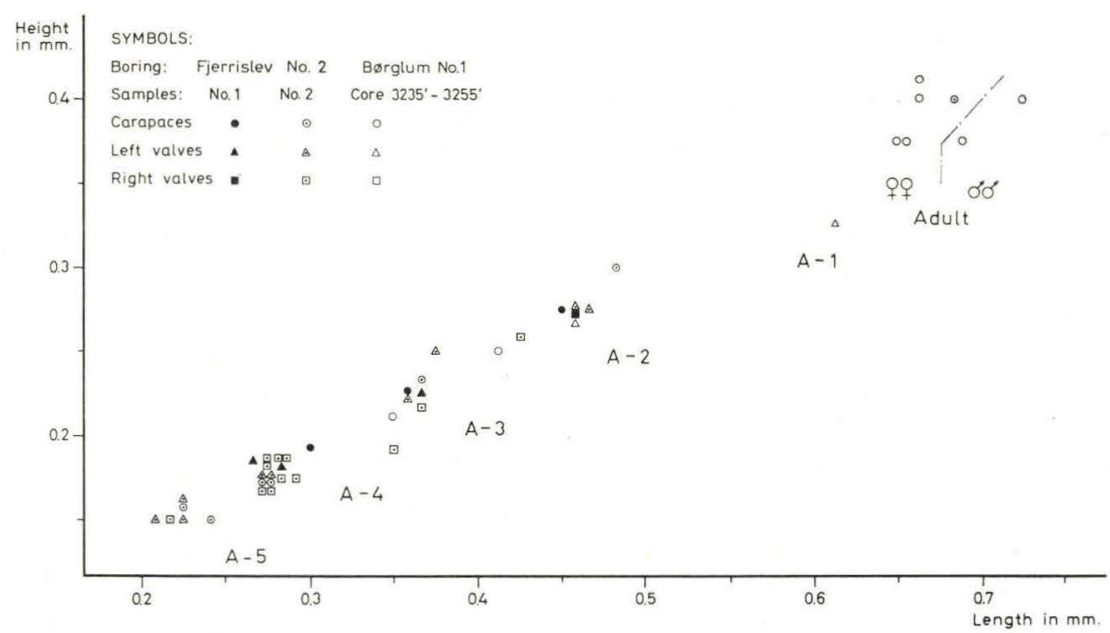

Fig. 9. Scattergram showing relations between numbers, lengths, and heights of Mandelstamia (Xeromandelstamia) tumida nov. sp. in three samples. Fjerritslev No. 2 with samples No. 1 and No. 2 are the uppermost sample and the type sample from core $474-480 \mathrm{~m}$. 


\section{Distribution}

NW. Danish Embayment: Børglum and Frederikshavn Formation, Lower? and Upper Kimmeridgian.

DoRset: Lowermost G. spinosa Zone, Upper Kimmeridgian. Kimmeridge Clay.

\section{? Subfamilia Paradoxostominae Brady and Norman 1889 \\ Genus Aaleniella PLumhoff 1963}

\section{Diagnosis}

Small, elongated, and laterally compressed species with straight dorsal margin. The left valve overlaps the right valve along the anterior, ventral, and posterior margins. Along the dorsal margin the right valve comes up over the dorsal margin of the left valve, and this overlap is especially distinct at the anterior cardinal angle. The adductor scars consist of four small, close-set scars arranged in a gently curved ventrodorsal line. Anterodorsally of this is a round antennal scar. The marginal zones are of median width with well developed vestibules, and with few and straight radial pore canals. The hinge in the right valve consists of a smooth often weakly developed furrow and two smooth, elongated, and not well developed terminal teeth.

\section{Subgenus Aaleniella (Aaleniella) Plumhoff 1963}

Type Species. Aaleniella (Aaleniella) compressa Plumhoff 1963.

\section{Diagnosis}

Small, smooth species of the genus Aaleniella with long and straight dorsal margin and rounded posterior end. Anteroventrally of the adductor scars two mandibular scars can be observed.

\section{Remarks}

Only two species have so far been included in this genus (the type species and Aaleniella sp. 1 Plumhoff 1963), and both are from the lower part of the Middle Jurassic in Germany. Aaleniella sp. 1 has not such strongly converging dorsal and ventral margins as the type species.

\section{Subgenus Aaleniella (Danocythere) nov.}

Type Species. Aaleniella (Danocythere) gracilis nov. sp.

Derivation of Name. Dano- is a short, clear, and specific prefix to the common generic name-cythere. 


\section{Diagnosis}

Species of the genus Aaleniella with a relatively short and straight dorsal margin. The posterior end is pointed and somewhat drawn out. The vestibule in the anterior marginal zone is short and deep.

\section{Remarks}

Mandibular scars have not been seen in the material.

The exterior features of the genus Pyrocytheridea Luubimova 1955 are very similar to Aaleniella, especially the larval stages of this species, but it has a different and more complicated hinge. In the species of Pyrocytheridea vestibules have not been demonstrated.

The larvae of the species of $A$. (Danocythere) show in the exterior features remarkable similarities to the adult specimens of the older subgenus A. (Aaleniella).

Aaleniella (Danocythere) gracilis nov. subgen. et sp.

Plate IV figs. 2a-f; textfig. 10.

1969 ?Pyrocytheridea sp. KILENYI, p. 33, pl. 27 figs. 25-26.

(MS) Aaleniella (Danocythere) gracilis BRUUN CHRISTENSEN and KILENYI - BRUUN CHRISTENSEN (MS).

Derivation of Name. Of Latin gracilis, slender.

Holotype. Right valve. Plate IV fig. 2a textfig. 10. D.G.U. Catalogue No. 1970 $\mathrm{OBC}-34$.

Type Locality. Borehole Fjerritslev No. 1, D.G.U. File No. 24.216.

Type Stratum. Dark claystone $474-480 \mathrm{~m}, 147 \mathrm{~cm}$ below the top of the core. Børglum Formation. Upper Kimmeridgian.

Material in the Sample. 19 carapaces and five valves.

\section{Diagnosis}

A small, smooth species of Aaleniella (Danocythere) with flattened lateral surfaces. The posterior terminal process is pointed and somewhat drawn out.

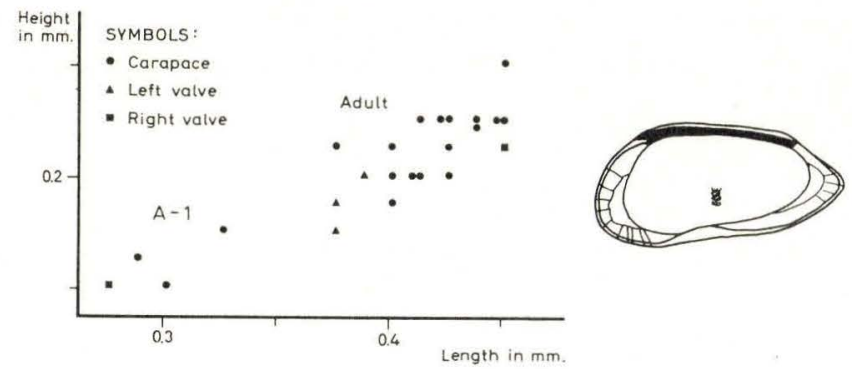

Fig. 10. Scattergram showing relations between numbers, lengths, and heights of Aaleniella (Danocythere) gracilis nov. subgen. et sp. from the type stratum. Outline of the holotype is figured. The widths of adult carapaces are $0.15-0.19 \mathrm{~mm}$. 


\section{Description}

In lateral view the carapace is oviform, with pointed ends. The dorsal margin is straight, the anterior cardinal angle is weakly developed and is situated one-fourth of the valve's length from the anterior. The posterior cardinal angle is one-fifth of the valve's length from the posterior. The posterior margin is symmetrically rounded in an even curve, just below the midheight of the valve. The anterior margin joins smoothly the nearly straight ventral margin, which continues in a slightly convex form to join the posterior end. The posterodorsal margin is either slightly concave or more commonly straight or slightly convex.

In dorsal view the carapace is gently arched and the central portions are nearly parallel. At a distance of one-sixths of the carapace's length from the anterior and posterior the sides of the valve begin to converge strongly. In frontal view the greatest breadth of the carapace is between one-third and half of the height from the ventral and the lateral surfaces of the valves meet in a right angle at the dorsal margin. In cross section the ventral part of the carapace varies from the anterior to the posterior. In the anterior part the outline is somewhat pointed, at midway it is semicircular and in the posterior half the lateroventral surfaces extend below the ventral margin.

The left valve is larger than the right with strong overlap along the anterior, ventral and posterior margins. Along the straight dorsal margin the periphery of the right valve is raised above that of the left.

The valve surface is smooth and shiny.

The adductor muscle scars consist of four extremely small scars arranged in a near vertical row. In front of them is a large, rounded antennal muscle scar. The normal pore canals are spread irregularly over the valve and seem to be present in relatively small number.

Eye spots have not been observed. The marginal zone is relatively broad. The zone of concrescence has about the same width along its entire length. A deep vestibule is developed in the middle of the anterior and posterior marginal zone, from which the irregularly distributed radial pore canals radiate outwards. These are straight, numbering 10-12 along the anterior and 4-5 along the posterior margin.

The hinge in the left valve consists of a long smooth median bar, the ends of which are bent slightly downwards. Two terminal sockets, smooth and elongated, are situated above the ends of the median bar. These sockets are open towards the dorsal margin to receive corresponding teeth from the right valve. The median hinge element of the right valve consists of a long, smooth bar, which is in a dorsal position compared with the median bar of the left valve. The terminal teeth in this valve are separated from the median bar by a shallow groove which opens outwards to the dorsal margin. The terminal teeth are the continuation of the selvage and are bent slightly inwards, parallel with the median bar. 


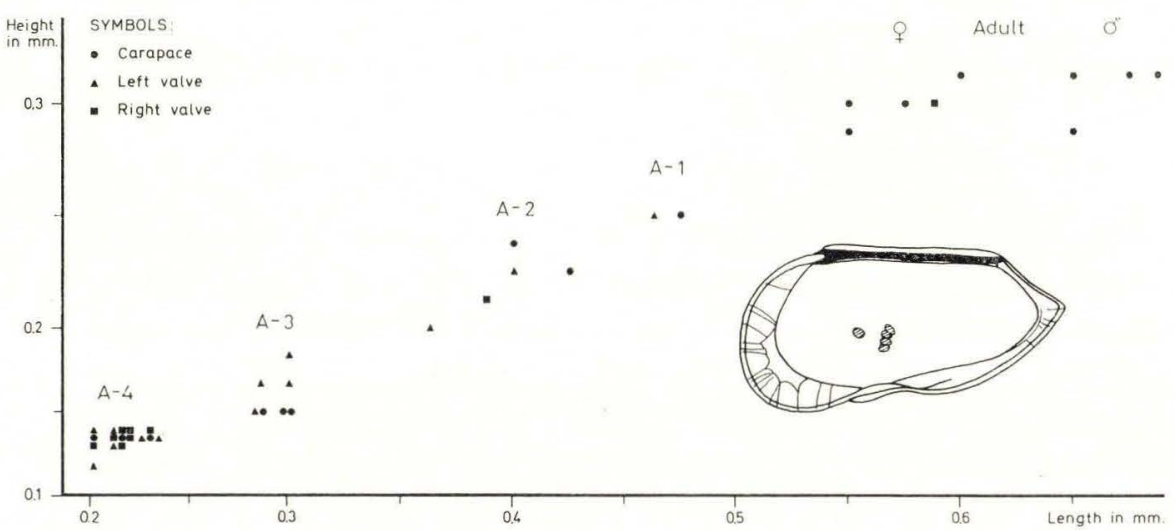

Fig. 11. Scattergram showing relations between numbers, lengths, and heights of Aaleniella (Danocythere) inornata (KILENYI) from boring Fjerritslev No. 1, core $474-480 \mathrm{~m}$. Outline of the adult right valve is figured. The widths of adult females and males are $0.18-0.20 \mathrm{~mm}$ and $0.19-0.20 \mathrm{~mm}$.

Juvenile valves have narrow marginal zones. Dimorphism has only been observed in the adult stage.

Relatively few of the adult specimens show a pointed and drawn out posterior end. This characteristics is more clearly seen in the right valve, where the posterodorsal margin can be weakly concave. All three single left valves and most of the carapaces in the material from Denmark have convex posterodorsal margins.

\section{Distribution}

England: Dorset, Upper Kimmeridge Clay, Zone of Galliaecytheridea spinosa KilenYI, and Cumnor Hill, near Oxford.

NW. Danish Embayment: Børglum and Frederikshavn Formation. Upper Kimmeridgian.

\section{Aaleniella (Danocythere) inornata (KILENYI 1969)}

Plate IV figs. 3a-g; textfig. 11.

1969 ? Acrocythere inornata KILENYI, p. 144, pl. 28 figs. 40-43.

(MS) Aaleniella (Danocythere) inornata (KILENYI) - BRUUn CHRISTENSEN (MS).

\section{Diagnosis}

A large and reticulate species of the subspecies Aaleniella (Danocythere) characterised by longitudinal lists in the central part of the lateral valve surface. 


\section{Remarks}

The name of the species is misleading. Originally the name of ornata was suggested for the Danish material, as opposed to two smooth species (Bruun Christensen MS), but the type material from Dorset was so poorly preserved, that special methods of colouring had to be used before the reticulation was recognized.

The species shows great exterior similarities to Palaeocytheridea bakirovi Mandelstam 1947 from the Middle Jurassic deposits of Mangeschlak in the Caspian Sea. These exterior similarities are greater that to P. parabakirovi MaLz 1962 from Middle Callovian in Northwest Germany. Both of these species have dentate hinge elements and no vestibules (cf. MaLz 1959, 1962).

\section{Distribution}

DORSET: Galliaecytheridea sp.nosa Zone, Upper Kimmeridge Clay.

NW. Danish Embayment: Børglum and Frederikshavn Formations, Upper Kimmeridgian.

\section{Familia Progonocytheridea Sylvester-Bradley 1948}

Genus Rectocythere MaLz 1958

Subgenus Rectocythere (Rectocythere) MaLz 1958

Type Species. Rectocythere (Rectocythere) iuglandiformis (KLINGLER 1955).

\section{Diagnosis}

Species of the genus Rectocythere without lateroventral ridge or alae. The marginal zone without vestibules as inner margin and the line of concrescence coincidence.

\section{Subgenus Rectocythere (Lydicythere) nov.}

Type Species. Rectocythere (Lydicythere) scandia nov. sp.

Derivation of Name. Lydi- is a short, clear and specific prefix to the common generic name -cythere.

\section{Diagnosis}

Small species of the genus Rectocythere with more or less irregular knobs on the lateral surfaces, with a vertical median sulcus along nearly the entire height of the valve, and a lateroventral ridge with ala-like structure of knobs. Inner margin and the line of concrescence do not coincide, and there are narrow vestibules along the marginal zones. 
Remarks

Malz (1966, p. 408) mentioned that "indet. gen. sp. 1" Oertli, Brotzen, and BARTENSTEIN 1961 has to be included in Rectocythere on the basis of the general outline and the number of marginal pore canals. Further studies of this species $R$. (Lydicythere) scandia nov. sp. and other species from the Danish Embayment have supported such a view. Moreover, the species Klieana? pustulata Klingler 1955 has to be included on the same basis in the genus. An English species (indet. gen. A. sp. 1 Kilenyi 1969, p. 137, pl. 31, fig. 20-21) could well be a species of Rectocythere (Lydicythere) nov. subgen. The species Mandelstamia verrucifera LJUBIMOVA 1955 from Oxfordian of U.S.S.R. is difficult to include in the subgenus because of the incomplete description given by LJuBimova (1955).

All the species of $R$. (Lydicythere) nov. subgen. have so far been found in deposits of Lower Kimmeridgian Age. The, as yet unsubstantiated, occurrences in Upper Oxfordian and Upper Kimmeridgian deposits may reduce the biostratigraphical value of the new subgenus.

\section{Rectocythere (Lydicythere) scandia nov. sp. Plate IV figs. 4a-f.}

1961 Indet. gen. sp. 1 Oertli, Brotzen \& Bartenstein, p. 16, plate 1 figs. 7-14.

1958 Rectocythere (n. subgen.) aff. R. pustulata (KLINGLeR) - BruUn Christensen, text fig. 1, fig. 3 .

(MS) Rectocythere (Lydicythere) scandia BRUUN CHRISTENSEN \& KILENYI - BRUUN CHRISTENSEN (MS).

Derivation of Name. Referring to the region of occurrence. The species has been found in Denmark and Sweden in the Fennoscandian Border Zone.

Holotype. A left valve, plate IV fig. 4c. D.G.U. Catalogue No. 1970 - OBC - 46. Type Locality. The pit of Fyleverken, Fyledalen, Scania.

Type Stratum. Ostracod Zone I: Light greenish, greasy clay, lower part of the Fyledal Clay. Lower Kimmeridgian.

Material in Sample. 28 valves from the type stratum.

Diagnosis

A small species of Rectocythere (Lydicythere) with more or less inflated knobs, irregularly spaced on the lateral valve surfaces. Between the knobs the surface varies from smooth to finely granular or very weakly reticulate. The posterior part of the ventral rib is composed of a number of closely spaced knobs.

\section{Affinities}

One of the authors (O.B.C.) sent specimens of this species from Fyledalen in Scania to Dr. Malz (Forschung-Institut Senckenberg, Frankfurt a. M.) who compared these with specimens of Rectocythere (Lydicythere) pustu- 
lata (KLingler 1955). Dr. Malz informs us (personal communication to O.B.C. 7.4.1967) that the two species are closely related, but can be separated on the basis of size and by the fact that the knobs on the valve surface are more conspicuous in Rectocythere (L.) pustulata and by the more pointed posterior end of Rectocythere (L.) scandia. Dr. MALz remarks that the ventral rib, the characteristic feature of the subgenus, is present in Rectocythere (L.) pustulata (KLINGLER). This has not been mentioned or illustrated by KLINGLER, who only shows elongated knobs in a row along the ventral margin (1955, plate 12, fig. 15a).

\section{Distribution}

NW. Polish Basin: Fyledal Clay, Lower Kimmeridgian. Scania.

NW. Danish Embayment: Børglum and Frederikshavn Formations, Lower Kimmeridgian. 


\section{DANSK SAMMENDRAG}

\section{OSTRACODEROG BIOSTRATIGRAFII NORD- OG VESTEUROPASKIMMERIDGIEN}

I Nordvesteuropa inddeles epoken $\varnothing$ vre jura almindeligvis i aldrene oxfordien, kimmeridgien og portlandien. Etagerne (aflejringerne), der er grundlaget for disse geologiske aldre, kan studeres i typeprofiler i Sydengland. Hver af de tre etager er yderligere underdelt kronostratigrafisk i $\varnothing v$ re og nedre afsnit på basis af lithologiske eller biostratigrafiske forhold i disse aflejringer.

Etagen kimmeridgien udgøres af den ca. 500 meter mægtige formation af Kimmeridge ler i Dorset. Denne formation er opdelt i talrige biostratigrafiske zoner ved hjælp af de fundne ammonitter (fig. 2 side 12), hvormed andre aflejringer med lignende ammonitfaunaer fra andre områder er blevet korreleret. Specielt har korrelationsmulighederne med nedre Kimmeridge ler været vellykket. Af flere årsager har dette medført, at navnet Kimmeridge de fleste steder udenfor Storbritannien oftest kun benyttes på aflejringer, der biostratigrafisk kan jævnføres med nedre Kimmeridge ler (nedre kimmeridgien). Dette har også tidligere været det mest almindelige i Danmark, hvor aflejringer, der kunne jævnføres med $\emptyset$ vre Kimmeridge ler som regel blev klassificeret som værende af nedre portlandien alder.

I nærværende arbejde benytter forfatterne navnet kimmeridgien i engelsk betydning og udstrækker etagen til helt at omfatte Kimmeridge ler. Portlandien knytter sig derfor udelukkende til de aflejringer (Portland Beds), der i Dorset overlejrer kimmeridgien (Kimmeridge Clay).

For nylig er et studium af ostracodfaunaerne fra Kimmeridge leret blevet publiceret (KILENYI 1969). Dette ligger til grund for nærværende arbejde, hvor stratotypen for kimmeridgien biostratigrafisk opdeles i fem tydelige ostracodzoner (fig. 2 side 12) på tilstedeværelsen og udviklingen af ostracodslægterne Galliaecytheridea og Mandelstamia. Et stort afsnit af etagen omfattende $\emptyset v r e$ del af nedre kimmeridgien og nedre del af фvre kimmeridgien samt mindre afsnit i $\varnothing$ vre kimmeridgien indeholder dog ikke ostracoder. På basis af denne ostracodzonering og det samlede indhold af ostracoder fra etagen gennemgås faunasammensætningerne i tilsvarende aflejringer i Nord- og Vesteuropa (fig. 1 side 9). Faunasammensætningerne er kendt fra litteraturen med undtagelse af faunaerne i det Danske Sænkningsområde, hvis sammensætning ikke tidligere har været publiceret (BRUUN 
CHRISTENSEN MS), og forhold vedrørende revisioner af typeprofilets ostracodarter meddeles.

Kapitlerne p. 14 og p. 18 indeholder en nærmere omtale af ostracodarterne fra de forskellige aflejringsområder, samt fra typeprofilet i Dorset. Zoneinddelingen af dette profil er ligeledes søgt genfundet i de andre områder.

Samtlige kendte ostracodarter af kimmeridgien alder i aflejringsområderne i Nord- og Vesteuropa er søgt opført i alfabetisk ordnede lister i kapitlet p. 26. Forfatterne har fors $\varnothing \mathrm{gt}$ at klassificere arternes stratigrafiske forekomster overvejende ved hjælp af litteraturen til nedre kimmeridgien (mrk. L i listerne) eller til $\varnothing$ vre kimmeridgien (mrk. U) fra de syv aflejringsområder. I listerne er der anført henvisninger til den mest betydningsfulde, nyere litteratur, hvor æeldre ostracodarter er blevet beskrevet. Sideangivelserne i listerne henviser til kapitlet p. 40 i nærværende arbejde, hvor nye og ældre arter er diagnosticeret og revideret. Samtlige anførte arter i de syv aflejringsområder er sammentalt med hensyn til optræden i $\emptyset$ vre og i nedre k'mmeridgien, samt i begge disse geologiske tidsafsnit i det pågældende område, samt antal arter der tid efter anden et eller flere steder optræder i begge disse geologiske tider. Ligeledes gøres der rede for områdernes fælles arter i antal og i procent ved anvendelse af en simpel binær korrelationskoefficient (Jaccards koefficient).

Det fundne talmateriale fremtræder delvis grafisk i fig. 4-7 (siderne 36-37). I fig. 5 er det samlede antal arter i de forskellige aflejringsområder fra nedre kimmeridgien indskrevet i cirkler på kortet. De i parentes anførte tal angiver antal arter heraf, der tillige forekommer i $\varnothing v$ re kimmeridgien i det pågældende område. Mellem de enkelte områder er der med tal opført antal af fælles arter i nedre kimmeridgien. Jaccards koefficient er angivet som procent af den samlede fauna mellem to områder ved antal linier indenfor multipla af fem procent.

I fig. 6 angiver tallene i cirklerne antal arter i de respektive områder, der tid efter anden er fundet både i nedre og $\emptyset$ vre kimmeridgien i Nord- og Vesteuropa. Tallene på enkelte forbindelseslinier mellem områderne angiver det antal arter, de pågældende områder har fælles af sådanne arter, der t:d efter anden er fundet $i$ aflejringer fra nedre og $\emptyset$ vre kimmeridgien.

Fig. 7 illustrerer ostracodfordelingerne i $\emptyset v r e$ kimmeridgien og svarer i opbygning til fig. 5 .

Af ovennævnte figurer fremgår det, at ostracodfaunaerne gennem nedre kimmeridgien som en helhed udviser et ensartet præg for Nord- og Vesteuropa. Det samme synes imidlertid ikke at være tilfældet med faunasammensætningen som helhed gennem $\emptyset$ vre kimmeridgien. Ved sammenligninger af fig. 6, fig. 7, listerne i kapitlet p. 26 og zoneundersøgelserne i kapitlerne p. 14 og p. 18 fremgår det, at der i øvre kimmeridgien fandtes to regioner med vidt forskellige ostracodfaunaer. 
Den ene region omfattede det Nordvest Danske Sænkningsområde og Dorset området. Dette synes at udgøre et sammenhængende Nords $\varnothing$ Bassin gennem yngste del af $\emptyset$ vre jura, idet artsfællesskab mellem områderne også kan påvises i portlandien (BRUUN CHRISTENSEN MS).

Den anden region omfattede det Nordvest Polske Bassin, hvori det Sydøst Danske Sænkningsområde naturligt indgår, desuden det Nordtyske Bassin, Pariser Bassinet og muligvis området i Sydvestfrankrig. Disse områder har forfatterne sammenfattet under betegnelsen den Midteuropæiske Region.

Forskellene i sammensætningerne af ostracodfaunaerne de to regioner imellem kan have flere årsager. Forfatterne forestiller sig imidlertid som den vigtigste årsag til dannelse af en faunabarriere mellem regionerne, at direkte marine forbindelser i $\varnothing \mathrm{vre}$ del af nedre kimmeridgien blev afbrudt f. eks. mellem Dorset området og Pariser Bassinet og mellem det Nordvest Danske Sænkningsområde og det Polske Bassin. Et marint og tilsyneladende langstrakt aflejringsbassin (Nords $\varnothing$ Bassinet) fra Dorset til den nordlige del af Jylland fortsatte således gennem hele kimmeridgien (og i portlandien) tilsyneladende med en relativt ensartet ostracodfauna.

Disse regionale og stratigrafiske forhold bliver belyst i kapitlet p. 35, hvor den stratigrafiske betydning af ostracodslægterne Mandelstamia og Galliaecytheridea i Nords $\varnothing$ Bassinet fremhæves. D:sse slægters tilstedeværelse og andre forhold i ostracodfaunaerne i Nords $\varnothing$ Bassinet viser slægtskab med ostracodfaunaer beskrevet fra Volga-Ural området i Sovjetunionen. I dette område findes stratotypen for volgien etagen, hvis nedre dele er af kimmeridgien alder. Imidlertid er korrelationer de to områder imellem meget vanskelige på grund af de store afstande, de svage biostratigrafiske korrelationsmuligheder og manglen på kronostratigrafiske korrelationsindikatorer. De sidstnævnte skal nok snarere søges blandt fysiske end blandt biologiske faktorer i aflejringerne. Muligheden for, at der har eksisteret et fra verdenshavet afskilt $\varnothing v r e$ jurassisk, borealt indhav (HALLAM 1969) med specielle aflejringsforhold forøger udsigten til, at fysiske forandringer i dette indhav ikke kun forårsager de ret ukontrollable biologiske forandringer, men tid efter anden forårsager sådanne forandringer, at disse kan aflæses direkte i aflejringerne forskellige steder i et sådant indhav og anvendes i den kronostratigrafiske korrelation.

Kapitlet p. 40 indeholder oprettelse af syv nye arter og to nye underslægter. Ialt er 19 arter anført med diagnoser, synonymlister og redeg ørelser for stratigrafisk udbredelse. De anførte arter har alle tidligere været publiceret under forskellige navne, som det fremgår af synonymlisterne til hver art. Af stor vigtighed har det imidlertid været at klarlægge forholdene omkring hanner og hunner hos forskellige ostracodslægter, idet blandt andet de to køn har været beskrevet som forskellige arter. 


\section{REFERENCES}

Arkell, W. J., 1933. The Jurassic System in Great Britain. - 681 pp., 41 pl. Oxford.

- 1947. The Geology of the Country around Weymouth, Swanage, Corfe and Lulworth. - Mem. geol. Surv. G. B. 386 pp.

- 1956. Jurassic Geology of the World. - 806 pp., 41 pl. Edinburgh.

Barker, D., 1966 a. Ostracods from the Portland Beds of Dorset. - Bull. Brit. Mus. (Nat. Hist.), 11, 9, pp. 447-457, pls. 1-6. London.

- 1966 b. Ostracods from Portland and Purbeck Beds of Aylesbury District - Bull. Brit. Mus. (Nat. Hist.), 11, 9, pp. 459-487, pls. 7-9. London.

BieleckA, W. and STYK, O. 1963. Mikropaläontologische Charakteristik des Malms im westlichen und östlichen Teil des Pommerschen Parantiklinoriums. - Ber. Geol. Gesellsch., 8, pp. 409-422, 1 fig., 3 tab. Berlin.

- 1964. Stratygrafia mikropaleontologiczna jura górnej w wierceniach Kcynia I, II, IV - Inst. Geol., Biul. 175, pp. 147-152, 3 plates. (English Summary: Micropalaeontological Upper Jurassic Stratigraphy in the Kcynia I, II and IV Bore-Holes) Warszawa.

- 1968. Analiza zespołów mikrofauny oksfordu i Kimerydo Polski niżowej w zalezńosci od różnic facjalnych - Kwarst. Geol., 2, pp. 324-344, 8 figs., 1 tab. (English Summary: Distribution of Oxfordian and Kimmeridgian Microfauna Assemblages in the Lowland Area of Poland, depending upon Facies Differences), Warszawa.

Bischoff, G. and Wolburg, J., 1963. Zur Entwicklung des Ober-Malm im Emsland Erdoel-Zeitschrift, 10, pp. 445-472. Wien.

Casey, R. 1967. The Position of the Middle Volgian in the English Jurassic. - Proc. Geol. Soc. London, 1640, pp. 128-133, London.

Christensen, O. Bruun, 1965. The Ostracod Genus Dicrorygma Poag 1962 from Upper Jurassic and Lower Cretaceous. - Geol. Surv. Denmark, II, 90, pp. 23, 2 figs., 2 plates. København.

- 1968 (1965). Biostratigrafiske undersøgelser af Øresundsboringer overvejende på grundlag af ostracoderne (English Summary: Biostratigraphical Investigations of the Pre-Quaternary in the Øresund Boreholes - Mainly on the Basis of Ostracods. In Larsen, G. and Christensen, O. Bruun, and Bang, I., and Buch, A. Øresund. Helsingør-Hälsingborg Linien. - Geol. Surv. Denmark, Report No. 1, pp. 90, 24 plates), pp. 53-62 and pp. 81-85, 1 plate. København.

- 1968. Some Deposits and Microfaunas from the Upper Jurassic in Scania. With New Species of Ostracods - Sveriges Geol. Undersökning, Ser. C, 632, pp. 46, 13 figs. Stockholm.

- 1970. Marine Communications through the Danish Embayment during Uppermost Jurassic and Lowermost Cretaceous - Geoscience and Man (in print).

- (MS) Biostratigraphical and Paleontological Studies of Ostracod Faunas from the Upper Jurassic and the Lower Cretaceous in the Danish Embayment. - Geol. Surv. Denmark (In preparation). 
Cope, J. C. W., 1967. The palaeontology and stratigraphy of the lower part of the Upper Kimmeridge Clay. - Bull. Brit. Mus. (Nat. Hist.), 15,1, pp. 1-79. London.

- 1968. Propectinatites, a new Lower Kimmeridgian ammonite genus. - Palaeontology, 11, pp. 16-18. London.

- and Hallam, A., and Torrens, H. S. et al., 1969. International Field Symposium on the British Jurassic. Excursion No. 1, Guide for Dorset and South Somerset Geology Department, Keele University.

Donze, P., 1960. Les formations du Jurassique terminal dans la partie nord-ouest de l'île d'Oléron (Charente - Maritime) - Annls. Univ. Lyon, C 12, pp. 5-30. Lyon.

FitTon, W. H., 1836. Observations on some of the strata between the Chalk and Oxford Oolite in the South-East of England. - Trans. Geol. Soc. London, 2, 4, pp. 103-388. London.

GlashofF, H., 1964. Ostrakoden - Faunen und Paläogeographie im Oxford NWEuropas - Paläont. Zeit., 38, 1/2, pp. 28-65, pl. 4-5. Stuttgart.

GUYADER, J., 1962. Reconnaissance geólogique de la zone portuaire Hauraise. - Bull. Soc. Géol. Normandie, 52, pp. 21-29.

Hallam, A., 1969. Faunal Realms and Facies in the Jurassic Palaeontology, 12,1, pp. 1-18, 4 figs. London.

KILENYI, T. I., 1955. Oertliana, a new ostracod genus from the Upper Jurassic of North-West Europe. - Paleontology, 8, 3, pp. 572-576, pl. 79. London.

- 1969. The Ostracoda of the Dorset Kimmeridge Clay. - Palaeontology, 12,1, pp. 112-160, 9 figs., pls. 23-31. London.

KLINGLER, W., 1955. Mikrofaunistische und stratigraphisch-fazielle Untersuchungen im Kimmeridge und Portland des Weser-Aller Gebietes. - Geol. Jb., 70, pp. 167246, 7 figs., pls. 6-22. Hannover.

- 1956. Zur Gliederung des Oberen Malm in Nordwestdeutschland - Erdöl und Kohle, 9, pp. 578-579.

- and Malz, H., and Martin, G. P. R. 1962. Malm NW-Deutschlands (in Leitfossilien der Mikropaläontologie, pp. 159-190, tab. 10, pl. 22-27, fig. 15). Berlin.

Larsen, G., 1966. Rhaetic - Jurassic - Lower Cretaceous Sediments in the Danish Embayment. - Geol. Surv. Denmark, II, 91, pp. 127, 17 pl. København.

Любимова, П. С., 1955. Остракоды мезозойских отложении́ Среднего Поволжья и Обшего Сырта. - Тр. ВНИГРИ, 84, pp. 1-191, Leningrad-Moscow.

- 1958. Триасовые и юрские остракоды восточных районов Украины. - Микрофауна, VIII, 34, pp. 534-583, 3 pls. Leningrad.

Malz, H., 1957. Macrodentina maculata n. sp., ein stratigraphisch wichtige Ostracod im Oberen Malm. - Senck.leth., 38, 3/4, p. 250. Frankfurt a. M.

- 1958 a. Nodophthalmocythere n. gen. (Ostrac., Ob. Jura) nebst einer Abgrenzung gegen ähnliche Gattungen - Senck.leth., 39, 1/2, pp. 119-133. Frankfurt a. M.

- 1958 b. Die Gattung Macrodentina und einige andere Ostracoden - Arten aus dem Oberen Jura von NW-Deutschland, England und Frankreich. - Abh. senckenb. naturf. Ges., 497, pp. 1-67, 11 pl., 3 tab., Frankfurt a. M.

- 1959. Nomenklatorisches zu einigen Ostracoden-Einheiten. - Senck.leth., 40, 5/6, pp. 409-413. Frankfurt a. M.

- 1962. Palaeocytheridea im oberen Dogger NW-Deutschlands. - Senck.leth., 43, 3, pp. 235-241. Frankfurt a. M.

- 1966. Rectocythere rugosa, eine neue Ostracoden-Art aus dem französischen Portlandien. - Senck.leth, 43, 4, pp. 405-409, 9 figs. Frankfurt a. M.

Мандельштам, М. И., 1947. Ostracoda из отложений средней юры лолуострова Мангышлака. Тр. ВНИГРИ, pp. 239-262, 2 pls. Leningrad-Moscow. 
Martin, C. P. R., 1940. Ostracoden des Norddeutschen Purbeck und Wealden Senckenb., 22 pp. 275-361, 13 pls. Frankfurt a. M.

- 1958. Cetacella, eine neue Ostracoden-Gattung aus dem Kimmeridge Nordwestdeutschlands. - Paläont. Zeitsch., 32, pp. 190-196. Stuttgart.

- and WeIler, W., 1957. Das Aldorfer Otolithen-»Pflaster« mit seine Fauna. Senckenb. leth., 38, 3/4, pp. 211-250, 3 pl. Frankfurt a. M.

Neale, John W., and Kilenyi, T. I., 1961. New species of Mandelstamia (Ostracoda) from the English Mesozoic. - Palaeontology, 3, 4, pp. 439-449. London.

Oertir, H. J., 1957. Ostracodes du Jurassique supérieur du Bassin de Paris (Sondage Vernon I) - Rev. Inst. franc. Pétrole, 12, pp. 647-695, pls. 1-7. Paris.

- 1959. Malm-Ostracoden aus dem schweizerischen Juragebirge. - Denkschr. schweiz. naturf. Ges., 83, pp. 1-44.

- 1963. Mesozoic Ostracod faunas of France - Leiden.

- and Brotzen, F. and BARTEnstein, H., 1961. Mikropaläontologisch-feinstratigraphische Untersuchung der Jura-Kreide-Grenzschichten in Südschweden. - Sveriges Geol. Undersökning, Ser. C, 579, pp. 3-24, 2 pls. Stockholm.

D’Orbigny, A., 1842-1849. Paléontologie française: Terrains jurassiques., Vol. 1. Paris.

Plumhoff, F., 1963. Die Ostracoden des Oberaalenium und tiefen Unterbajocium (Jura) des Grifhorner Troges, Nordwestdeutschland. - Abh. senckenb. naturf. Ges., 503, pp. 1-100. Frankfurt a. M.

Schмidт, G., 1954. Stratigraphisch wichtige Ostracoden im »Kimmeridge" und tiefsten »Portland " NW-Deutschlands. - Paläont. Zeitschr., 28, 1/2, pp. 81-101, pls. 5-8. Stuttgart.

- 1955. Stratigraphie und Mikrofauna des mittleren Malm im nordwestdeutschen Bergland mit einer Kartierung am südlichen Ith. - Abh. senck. naturf. Ges., 491, pp. 1-79, 2 fig., 18 taf., 1 map. Frankfurt a. M.

Steghaus, H., 1951. Ostracoden als Leitfossilien im Kimmeridge der Ölfelder Wietze und Fuhrberg bei Hannover. - Paläont. Zeitschr. 24, pp. 201-224. Stuttgart.

Steghaus, H., 1953. Über die Möglichkeit einer Gliederung des Weiß-Jura von Dalum - Ber. naturf. Ges. Freiburg, 43, 1, pp. 39-46, 1 fig., 2 pl. Freiburg.

Triebel, E., 1941. Zur Morphologie und Ökologie der fossilen Ostracoden. - Senck., 23 , pp. 294-400, 15 pls. Frankfurt a. M.

- 1954. Malm-Ostracoden mit amphidontem Schloß. - Senck. 1eth., 35, 1/2, pp. 3-16, 4 pls. Frankfurt a. M.

- and Bartenstern, H., 1938. Die Ostracoden des deutschen Jura. 1. Monoceratina Arten aus dem Lias und Dogger. - Senck., 20, pp. 502-518. Frankfurt a. M.

Webster, T., 1816. Picturesque Beauties of the Isle of Wight. London.

Wienholz, E., 1968. Ostracoden-faunaen der Jura/Kreide - Grenzschichten im Norden der Deutschen Demokratischen Republik. - Ber. deutsch. Ges. geol. Wiss., A., Geol. Paläont., 13, 2, pp. 233-238. Berlin.

Ziegler, B., 1967. Die Ammoniten - Gattung Aulacostephanus im Oberjura - Paläontographica, 119A, pp. 1-172, 22 pl. Stuttgart. 
PLATES 
Plate I

Magnifications: $50 \times$

D.G.U.

Catalogue Text

No.

Figs. 1a-i Schuleridea (Schuleridea) moderata nov. sp. . . . . . . . . . . . . 41

1a, female carapace, right view, holotype ......... 1970-OBC-1

$1 \mathrm{~b}$, the same specimen in dorsal view.

1c, female carapace in right view, paratype ........ 1970-OBC-2

1d, female left valve, paratype .............. 1970-OBC-3

1e, larval carapace in right view, paratype ......... 1970-OBC-4

1f, female right valve ................... 1970-OBC-5

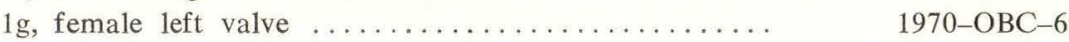

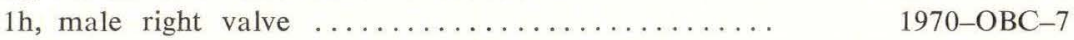

1i, male left valve ................... 1970-OBC-8

Boring Fjerritslev No. 1, core $474-480 \mathrm{~m}$.

Fig. 2a-f Gaillaecytheridea compressa nov. sp.

2a, female carapace, right view, holotype

(Brit. Mus. IO2763)

$2 \mathrm{~b}$, the same specimen in dorsal view.

2.c, male carapace, right view, paratype

(Brit. Mus. IO5013)

$2 \mathrm{~d}$, the same specimen in dorsal view.

Hounstout Cliff, Dorset.

2e, male carapace, right view $\ldots \ldots \ldots \ldots \ldots \ldots \ldots$

1970-OBC-9

2f, the same specimen, dorsal view.

Boring Fjerritslev No. 1, core $457-480 \mathrm{~m}$.

phot. Chr. Westergand (figs. 1a-b, 1h-i, 2e-f) - O. Neergaard Rasmussen (figs. $1 \mathrm{c}-\mathrm{g}, 2 \mathrm{a}-\mathrm{d})$

The specimens with numbers prefixed D.G.U. are in collection of the Geological Survey of Denmark. 
Plate I

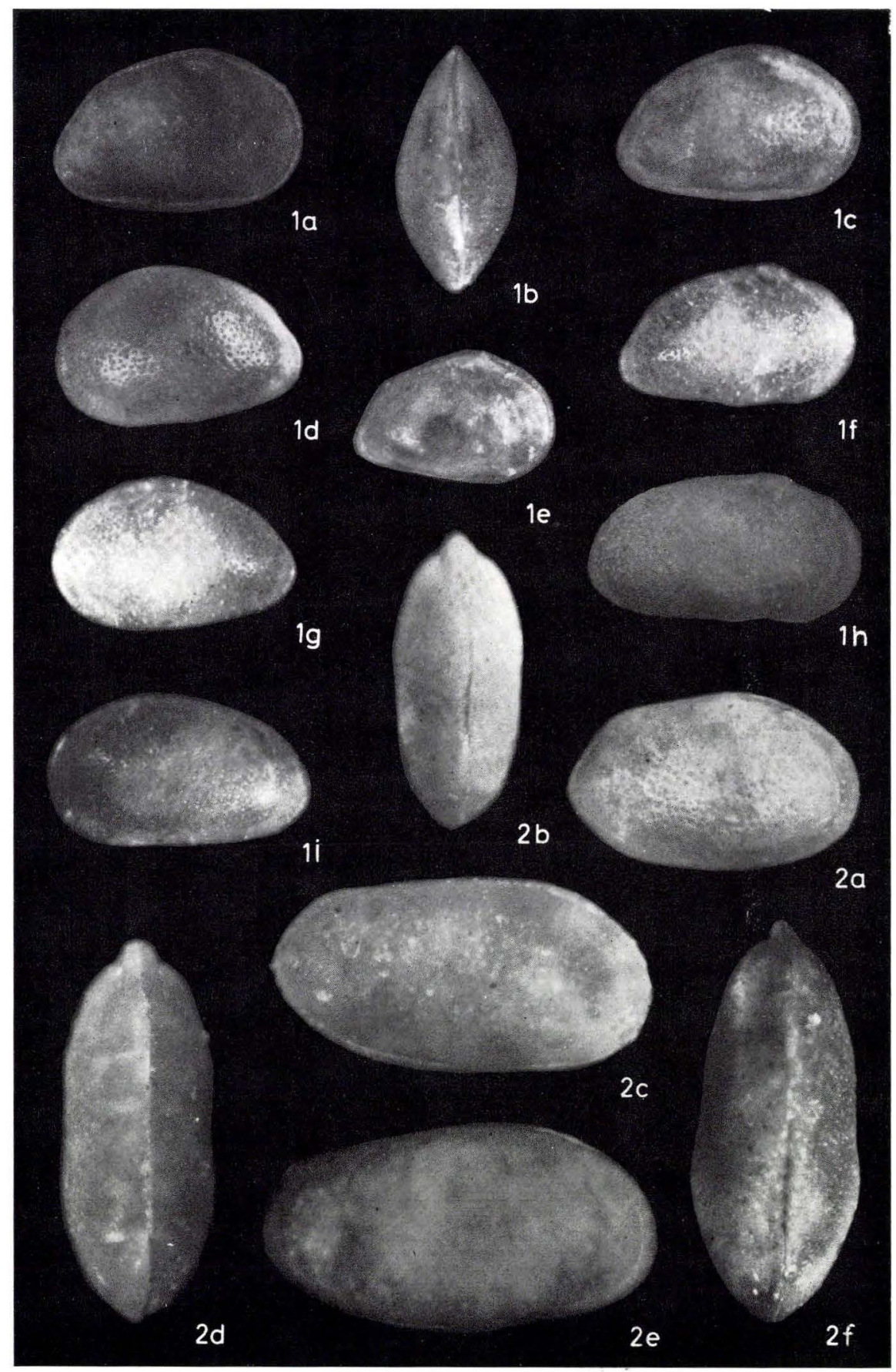


D.G.U.

Catalogue Text

No.

Figs. 1a-d Galliaecytheridea compressa nov. sp.

1a, female carapace, left view

1970-OBC-10

Upper Portland Sand, Isle of Portland

$1 \mathrm{~b}$, female carapace, left view

$1970-\mathrm{OBC}-11$

1c, the same specimen, dorsal view.

Boring Fjerritslev No. 2, 1079-1082 m

1d, female right valve, paratype

(Brit. Mus. IO5014)

Hounstout Cliff, Dorset.

Figs. 2a-b Galliaecytheridea gracilis GLASHOFF . . . . . . . . . . . . .

2a, left valve .......................... Univ. HU2.J.13)

G. dissimilis Zone, Kimmeridge Clay, Dorset.

2b, left valve .................... 1970-OBC-12

Boring Skagen No. 2, core 1315'-1325'

Figs. 3a-d Galliaecytheridea dorsetensis nov. sp.

3a, female carapace, right view .......... (Hull Univ. HU2.J.13.1)

$3 \mathrm{~b}$, the same specimen, dorsal view.

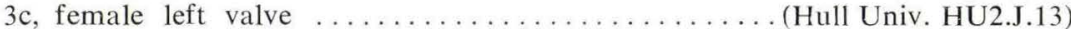

$3 \mathrm{~d}$, female right valve $\ldots \ldots \ldots \ldots \ldots \ldots \ldots \ldots \ldots \ldots$ (Hull Univ. HU2.J.13)

G. dissimilis Zone, Kimmeridge Clay, Dorset

Figs. 4a-b ? Galliaecytheridea dorsetensis nov. sp.

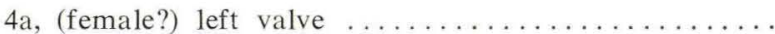

1970-OBC-13

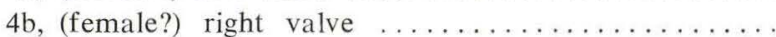

1970-OBC-14

Boring Skagen No. 2, core 1190'-1197'

Figs. 5a-b Galliaecytheridea minuta (SchmIDT) 44,49

5a, carapace, right view

1970-OBC-15

$5 \mathrm{~b}$, the same specimen in dorsal view.

Boring Skagen No. 2, core 1190'-1197'

phot. Chr. Westergand (figs. 1b-c, 4a-b, 5a-b) - O. Neergand Rasumssen (figs. 1a, 1d, 2a-b, 3a-d)

The specimens with numbers prefixed D.G.U. are in collection of the Geological Survey of Denmark. 


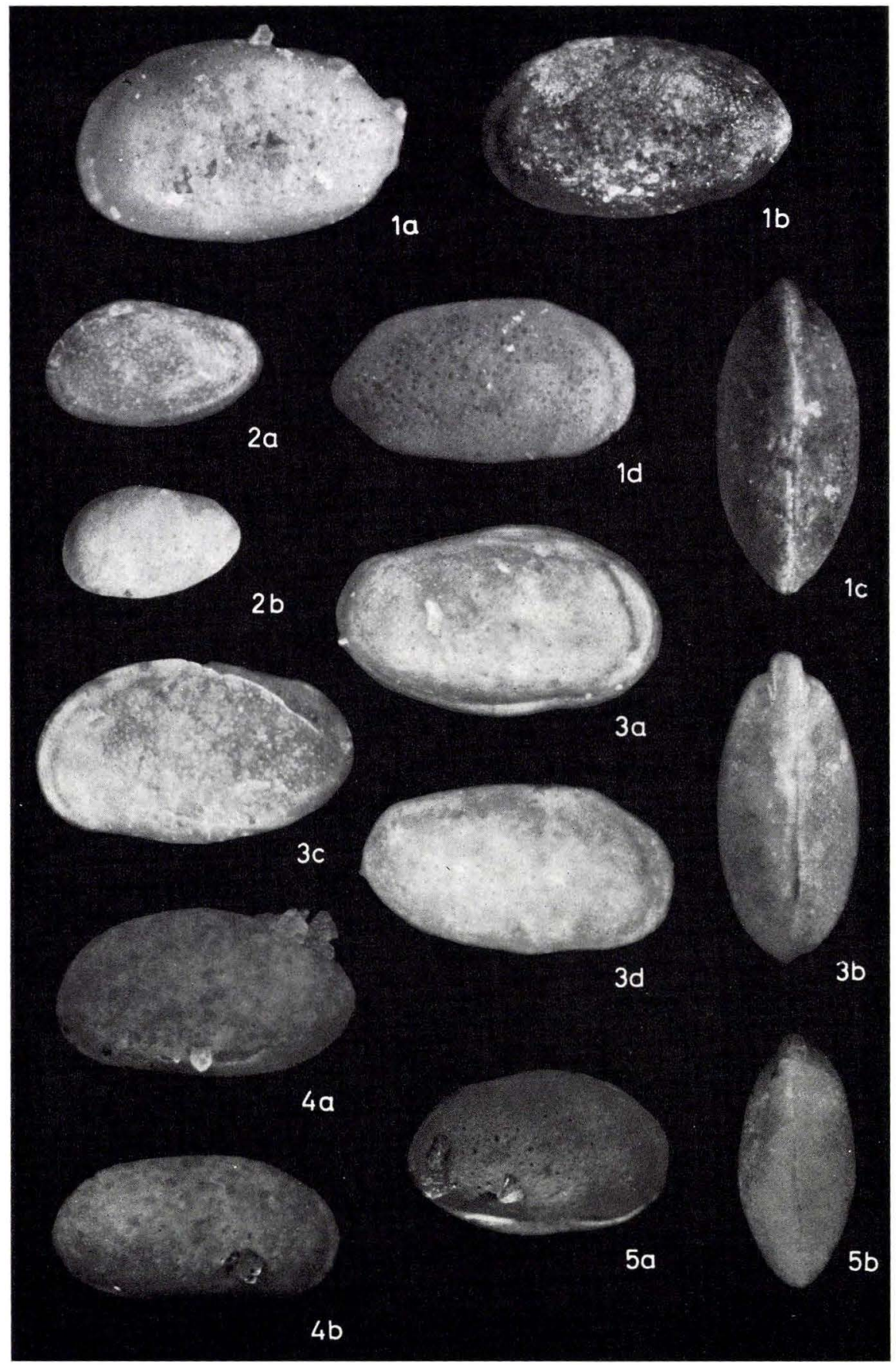


Figs. 1a-i Galliaecytheridea oertiii nov. sp.

D.G.U.

Catalogue Text

No.

1a, female left valve, paratype .

$1 \mathrm{~b}$, female carapace, right view, holotyp

1970-OBC-17

$1 \mathrm{c}$, the same specimen, dorsal view.

$1 \mathrm{~d}$, male carapace in right view, paratype

1970-OBC-18

1e, the same specimen, dorsal view.

1970-OBC-19

1f, larval right valve, paratype

1970-OBC-20

$1 \mathrm{~g}$, larval right valve, paratype $\ldots \ldots \ldots \ldots \ldots \ldots \ldots \ldots$

1970-OBC-21

1h, larval left valve, paratype $\ldots \ldots \ldots \ldots \ldots \ldots \ldots$

1970-OBC-22

1i, larval right valve, paratype $\ldots \ldots \ldots \ldots \ldots \ldots \ldots$

1970-OBC-23

Boring Øresund No. 1A, core $51.70-53.15 \mathrm{~m}$

Figs, 2a-c Galliaecytheridea spinosa KILENYI s. $l$.

$2 a$, right larval valve

$1970-$ OBC-24

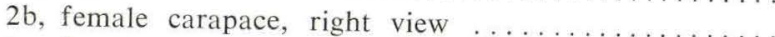

$1970-\mathrm{OBC}-25$

2c, female carapace, right view

1970-OBC-26

Boring Fjerritslev No. 1, core 474-480 m

Figs. 3a-f Mandelstamia (Xeromandelstamia) tumida nov. sp.

$3 \mathrm{a}$, left larval valve, paratype

1970-OBC-27

$3 \mathrm{~b}$, right larval valve

1970-OBC-28

Boring Fjerritslev No. 1, core 474-480 m

$3 \mathrm{c}$, larval carapace, right view

1970-OBC-29

$3 \mathrm{~d}$, the same specimen in dorsal view

Boring Børglum No. 1, core 3235'-3255'

3 e, female carapace, left view, holotype

3f, the same specimen, dorsal view.

1970-OBC-31

Boring Fjerritslev No. 1, core 474-480 m

phot. Chr. Westergand (figs. 1a-i, 2a-c, 3a, 3e-f) - O. Neergaard Rasmussen (figs. 3b-d)

The specimens with numbers prefixed D.G.U. are in collection of the Geological Survey of Denmark. 


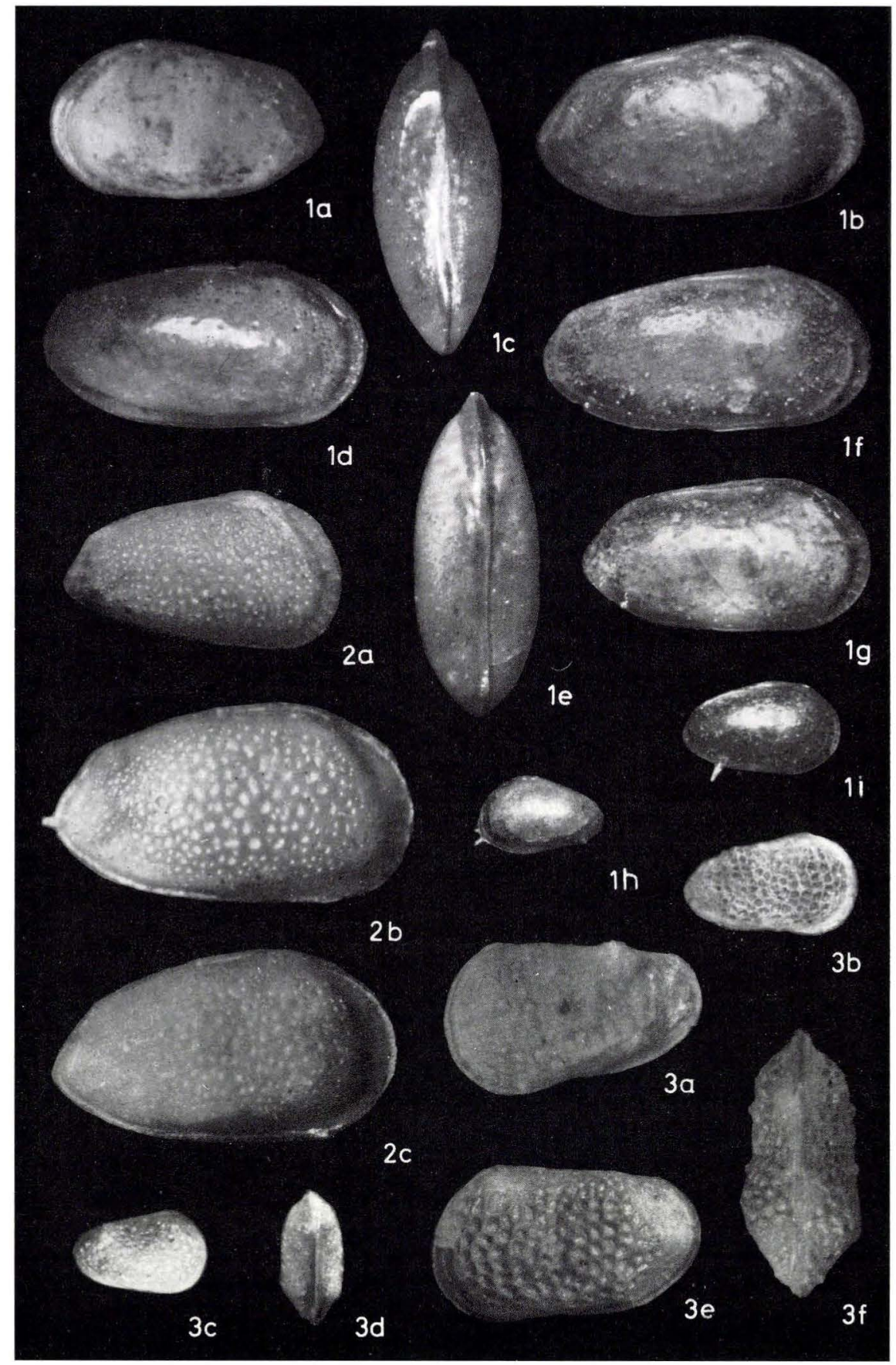


Figs. 1a-d Mandelstamia (Xeromandelstamia) tumida nov. sp.

1a, female carapace, right view

$1 \mathrm{~b}$, the same specimen, dorsal view.

1c, male carapace, right view $\ldots \ldots \ldots \ldots \ldots \ldots \ldots$

1970-OBC-33

$1 \mathrm{~d}$, the same specimen, dorsal view.

Boring Børglum No. 1, core $3235^{\prime}-3255^{\prime}$

Figs. 2a-f Aaleniella (Danocythere) gracilis nov. sp. et subgen.

$2 \mathrm{a}$, right valve, holotype

$2 b$, carapace, left view, paratype $\ldots \ldots \ldots \ldots \ldots \ldots$

1970-OBC-35

$2 \mathrm{c}$, the same specimen, dorsal view.

$2 \mathrm{~d}$, larval carapace, right view $\ldots \ldots \ldots \ldots \ldots \ldots \ldots$

$1970-\mathrm{OBC}-36$

2e, the same specimen, dorsal view.

2f, carapace, left view, paratype

1970-OBC-37

Boring Fjerritslev No. 1, core $474-480 \mathrm{~m}$

Figs. 3a-g Aaleniella (Danocythere) inornata (KILENYI)

$3 \mathrm{a}$, male carapace, right view

$1970-\mathrm{OBC}-38$

$3 \mathrm{~b}$, the same specimen, dorsal view.

$3 \mathrm{c}$, larval carapace, left view $\ldots \ldots \ldots \ldots \ldots \ldots \ldots$

1970-OBC-39

$3 \mathrm{~d}$, female carapace, right view $\ldots \ldots \ldots \ldots \ldots \ldots$

1970-OBC-40

3e, female carapace, right view $\ldots \ldots \ldots \ldots \ldots \ldots$

$1970-\mathrm{OBC}-41$

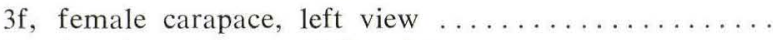

1970-OBC-42

$3 \mathrm{~g}$, female carapace, right view $\ldots \ldots \ldots \ldots \ldots \ldots$

$1970-\mathrm{OBC}-43$

Boring Fjerritslev No. 1, core 474-480 m

Figs. 4a-f Rectocythere (Lydicythere) scandia nov. sp. et subgen.

4a, left valve

$1970-\mathrm{OBC}-44$

Boring Skagen No. 2, core 1209'-1215'

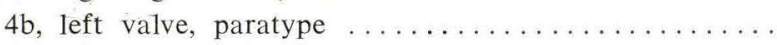

1970-OBC-45

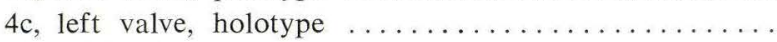

1970-OBC-46

$4 \mathrm{~d}$, right valve, paratype $\ldots \ldots \ldots \ldots \ldots \ldots \ldots \ldots$

1970-OBC-47

Ostracod Zone I, Fyleverken, Scania

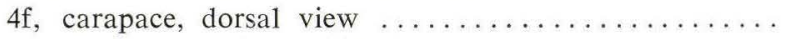

$1970-O B C-48$

$4 \mathrm{~g}$, the same specimen, left view.

Boring Skagen No. 2, core 1209'-1215'

phot. Chr. Westergatrd (figs. 2a-f, 3a-g, 4a, 4d-f) - O. NeergaArd Rasmussen (figs. 1a-d, 4b-c)

The specimens with numbers prefixed D.G.U. are in collection of the Geological Survey of Denmark. 


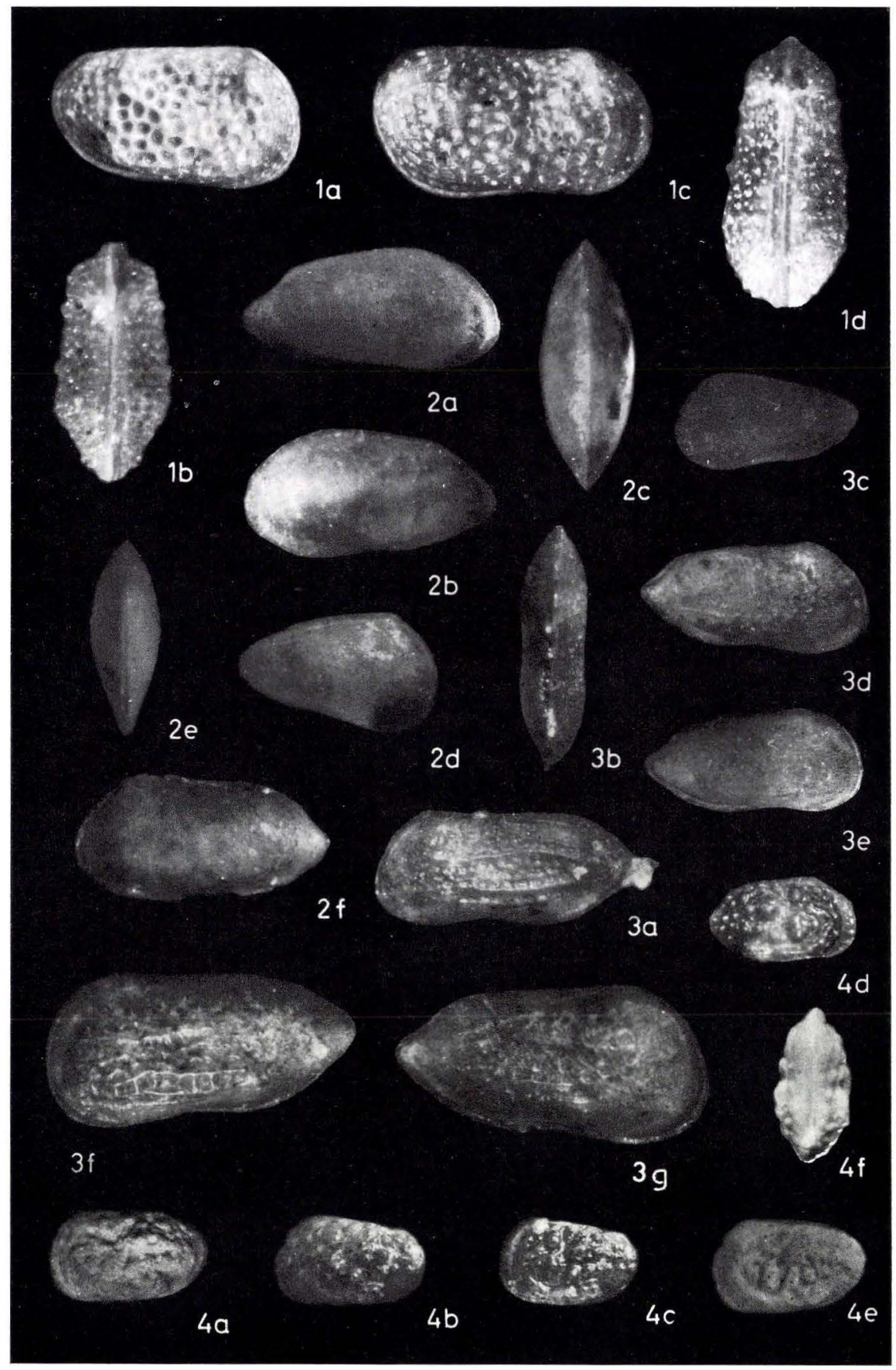

